\title{
The ETS Transcription Factor ERF controls the exit from the naïve pluripotent state
}

M. Vega-Sendino, ${ }^{1}$ T. Olbrich, ${ }^{1}$ D. Tillo, ${ }^{2}$ A. D. $T r a n,{ }^{3}$ C. N. Domingo, ${ }^{1}$ M. Franco, ${ }^{1}$ P. C. FitzGerald, ${ }^{4}$ M. J. Kruhlak, ${ }^{3}$ and S. Ruiz ${ }^{1, *}$

${ }^{1}$ Laboratory of Genome Integrity, ${ }^{2}$ Genetics Branch, ${ }^{3}$ Laboratory of Cancer Biology and Genetics and ${ }^{4}$ Genome Analysis Unit, CCR, NCI, NIH, Bethesda, MD, USA.

*Corresponding author: sergio.ruizmacias@nih.gov, @sruizmacias

The naïve epiblast undergoes a transition to a pluripotent primed state during embryo implantation. Despite the relevance of the FGF pathway during this period, little is known about the downstream effectors regulating this signaling. Here, we examined the molecular mechanisms coordinating the naïve to primed transition by using inducible ESC to genetically eliminate all RAS proteins. We show that differentiated RAS ${ }^{K O}$ ESC remain trapped in an intermediate state of pluripotency with naïve-associated features. Elimination of the transcription factor ERF overcomes the developmental blockage of RAS-deficient cells by naïve enhancer decommissioning. Mechanistically, ERF regulates NANOG expression and ensures naïve pluripotency by strengthening naïve transcription factor binding at ESC enhancers. Moreover, ERF negatively regulates the expression of the de novo methyltransferase DNMT3B, which participates in the extinction of the naïve transcriptional program. Collectively, we demonstrated an essential role for ERF controlling the exit from naïve pluripotency during the progression to primed pluripotency.

Teaser: ERF is the MAPK-dependent switch controlling the transition between naïve and primed pluripotency during embryonic development. 


\section{INTRODUCTION:}

31 Embryonic cells residing within the inner cell mass (ICM) are pluripotent as they have the

32 potential to generate all cell lineages of the organism. The state of pluripotency found in the

33 preimplantation epiblast, prior to any lineage specification, is usually defined as naïve (1).

34 However, as the embryo expands and develops after implantation, epiblast cells become

35 individually fated, although still retain pluripotent features. This state of pluripotency associated

36 to the post-implantation epiblast is defined as primed $(2,3)$. More than distinct pluripotent

37 states, naïve and primed can be viewed as different phases of a coordinated developmental

38 progression where naïve unbiased cells differentiate responding to inductive cues to initiate a

39 multi-lineage decision commitment at gastrulation $(2,3)$.

40 Naïve and primed pluripotent states can be captured in vitro by defined culture conditions.

41 Mouse embryonic stem cells (ESC) can be propagated in naïve conditions by using a combination

42 of a MEK inhibitor (PD0325091) plus a glycogen synthase kinase-3 inhibitor (CHIR99021;

43 hereafter referred as the $2 \mathrm{i}$ condition) (4). ESC grown under these conditions resemble embryonic cells residing in the pre-implantation embryonic (E) post-fertilization day E3.5-E4.25 embryo and have been extensively used to study the ground state of pluripotency. In addition, cultures of embryonic cells that retain primed pluripotency can also be established from post-implantation embryos (5). These epiblast stem cells (EpiSC) resemble transcriptionally the late epiblast layer of the post-implantation embryo at E6.0-E6.5. Both types of cells, naïve and primed, share the expression of core pluripotent transcription factors (TF), such as OCT4 and SOX2. However, 2iESC express specific naïve TF (REX1, NANOG or KLF4), which are absent in EpiSC, whereas EpiSC express epiblast specific genes (OCT6 or OTX2), absent in ESC. Importantly, these pluripotent cell types are interconvertible by modifying the culture conditions or expressing specific TF (3).

53 Recently, a distinct intermediate state of pluripotency has been identified, the rosette pluripotent state (6). Rosette embryonic stem cells (RSC) co-express naïve TF and the primed marker OTX2. Inhibition of WNT signaling drives the pluripotent transition from naïve to the rosette state while further activation of the RAS/MAPK signaling promotes the progression to

57 primed pluripotency (6). Furthermore, additional states of intermediate pluripotency 
59 (FSC) and chimera pluripotent stem cells (XPSC) $(7,8)$. These intermediates rely on exogenous

60 (XPSC) or autocrine (FSC) FGF signaling for their self-renewal in contrast to the requirements for

61 RSC. In summary, pluripotency can be considered as a dynamic property associated to different

62 stem cell states supported by defined pluripotent transcriptional networks.

63 Exit from naïve pluripotency during implantation is essential to fate epiblast cells residing in the

64 ICM with inductive signals prior to gastrulation. However, the molecular mechanisms

65 coordinating the naïve to primed transition are not fully understood. Moreover, despite the

66 relevance of the RAS pathway during this critical period, little is known about the downstream

67 effectors regulating the MAPK transcriptional program. We recently identified the transcriptional

68 repressor ERF, a member of the E26 transformation specific (ETS) family, as an important

69 regulator downstream of the RAS pathway in ESC (9). ERF is a TF that shuffles between the nuclei

70 and cytoplasm in ESC in a phosphorylation-dependent manner (10). In the absence of RAS/MAPK

71 signaling, ERF remains unphosphorylated and chromatin-bound in the nuclei while growth factor

72 stimulation keeps ERF inactive in the cytoplasm by ERK-dependent phosphorylation (10-12).

73 Importantly, the precise role of ERF during early embryonic development and pluripotent

74 transitions is unclear. Here, we show that ERF plays a dual role in the transition from naïve to

75 primed pluripotency. First, ERF binds to ESC super-enhancers to ensure optimal expression of

76 naïve TF in the absence of FGF signaling. Second, activation of MAPK signals induces the release

77 of chromatin-bound ERF, an event that is necessary and sufficient to trigger full commitment to

78 primed pluripotency. In addition, we also show that ERF controls the expression of the de novo

79 methyltransferase DNMT3B through LIN28 regulation, leading to the inactivation of the naïve

80 transcriptional program. Our data demonstrate that ERF is the MAPK-dependent switch that

81 controls the progression to primed pluripotency. 
RESULTS:

\section{ERF is expressed in the naïve pluripotent epiblast}

91 We first sought to determine the precise expression timing for ERF during embryonic

92 development. For this, we collected mouse embryos at different developmental stages and

93 performed immunofluorescence analyses. We determined that ERF expression peaks around

94 E3.5-E4.0 and is coincidental with the naïve pluripotent epiblast (Fig. 1A-C). ERF is expressed in

95 the naïve inner cell mass (ICM) and co-expressed with the naïve pluripotent markers NANOG and

$96 \mathrm{KLF} 4$ (Fig. 1B, C). In addition, we also detected ERF in the trophectoderm (TE), consistent with its

97 reported requirement for chorionic trophoblast differentiation (Fig. 1B, C) (13). Exit from naïve

98 pluripotency in vivo and downregulation of naïve associated markers strongly correlated with

99 decreased expression of ERF (Fig. 1B, C). We confirmed this expression pattern by analyzing

100 single-cell RNAseq datasets from embryos at different stages (fig. S1A) (14). These results showed

101 that ERF is upregulated during ICM formation and it is quickly downregulated before

102 implantation, suggesting a role for ERF in the naïve epiblast.

103 To validate our observations and assess the role of the MAPK pathway in ERF levels, we used an

104 ESC model deficient in H-RAS and N-RAS with a conditional knockout K-RAS triggered by the

105 addition of 4-hydroxytamoxifen (OHT) (RAS ${ }^{\text {lox/lox }}$ hereafter) $(9,15)$. These ESC allowed us to

106 generate cells devoid of all RAS proteins. We next applied a well-established in vitro protocol

107 using FGF2 and Activin-A (FA, hereafter) to differentiate naïve ESC, growing in 2i+LIF (2iL)

108 conditions, to post-implantation epiblast like stem cells (EpiLSC) and examined ERF levels (16,

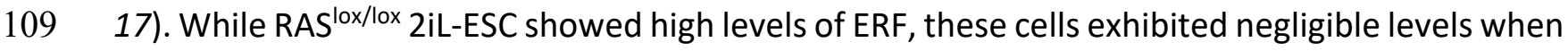

110 differentiated into EpiLSC (Fig. 1D). ERF is quickly phosphorylated upon differentiation and the

111 decrease in protein levels is due to transcriptional repression (fig. S1B) (18). We next examined

112 whether this downregulation depended on FGF/MAPK activation. Indeed, RAS-deficient cells

$113\left(\right.$ RAS $^{\mathrm{KO}}$ ) retained elevated levels of ERF after differentiation (Fig. 1D). Together, these results

114 pointed to a role for ERF in the exit from naïve pluripotency in a MAPK-dependent manner. 


\section{Downregulation of ERF is necessary for the successful exit from naïve pluripotency}

118 To evaluate the implication of ERF in the exit from naïve pluripotency, we examined its expression

119 along with markers of naïve pluripotency during the formation of embryonic rosettes. In this

120 assay, single cell suspensions of ESC embedded in Matrigel exit from naïve pluripotency and

121 develop into polarized rosettes that undergo lumenogenesis, mimicking the morphogenic events

122 of the epiblast (19). This in vitro system recapitulates the development of the ICM during peri-

123 implantation. Importantly, ESC rosettes generated under 2iL conditions lack lumen, continue to

124 express naïve markers, and eventually become disorganized over time (20). This showed that exit

125 from naïve pluripotency is necessary for successful polarization and lumenogenesis. Upon 2iL

126 removal and resuspension in Matrigel, RAS lox/lox rosettes showed polarization and lumen,

127 expression of the sialomucin protein podocalyxin (PDX) and downregulation of the naïve marker

128 NANOG (Fig. 2A-C and fig. S2A and B). However, RAS ${ }^{\mathrm{KO}}$ rosettes developed into a disorganized

129 group of cells, which lacked expression of PDX and retained naïve pluripotency markers (Fig. 2A-

$130 \mathrm{C}$ and fig. S2A and B). To test whether ERF regulates the exit from naïve pluripotency, we 131 generated ERF-knockouts $\left(\mathrm{ERF}^{\mathrm{KO}}\right)$ and evaluated rosette formation in all different genotypes

132 (RAS ${ }^{\text {lox } / l o x}, E R F^{\mathrm{KO}}, \mathrm{RAS}^{\mathrm{KO}}$, and $\mathrm{RAS}^{\mathrm{KO}} ; \mathrm{ERF}^{\mathrm{KO}}$ ). Elimination of ERF is sufficient to induce exit from 133 naïve pluripotency and to rescue the failed morphogenic events (polarization and 134 lumenogenesis) observed in RAS ${ }^{\mathrm{KO}}$ rosettes (Fig. 2A-C and fig. S2A and B). To further support 135 these observations, we generated reporter cell lines in our ESC by replacing the endogenous 136 coding sequence of the gene REX1 (also known as ZFP42) with a short half-life form of eGFP 137 (REX1-deGFP). Similar REX1-deGFP reporter ESC have been widely used as a faithful system to 138 monitor exit from naïve pluripotency (21). Induction of EpiLSC by FA in RAS ${ }^{\text {lox/lox } \text { and ERF }}$ ESO $^{\text {ESC }}$ 139 demonstrated efficient downregulation of the reporter while RAS ${ }^{K O}$ ESC showed no signs of 140 downregulation (Fig. 2D). However, exit from naïve pluripotency was efficiently achieved in $141 \mathrm{RAS}^{\mathrm{KO}}$; ERF${ }^{\mathrm{KO}}$ ESCS (Fig. 2D). Finally, we also examined the clonogenicity potential of RAS ${ }^{\mathrm{KO}}$; ERF $^{\mathrm{KO}}$ 142 ESC primed to exit naïve pluripotency. For this, cultures of ESC (RAS ${ }^{\text {lox/lox }}$, ERF $^{\mathrm{KO}}$, RAS ${ }^{\mathrm{KO}}$, and RAS ${ }^{\mathrm{KO}}$; $143 \mathrm{ERF}^{\mathrm{KO}}$ ) were withdrawn of $2 \mathrm{iL}$ for 2 days and plated back in 2iL conditions for a colony forming 144 assay. Cells that exit naïve pluripotency are irreversibly committed and have lost the ability to 145 generate colonies in 2iL conditions. Indeed, while RAS ${ }^{\mathrm{KO}}$ ESC were able to generate alkaline 
146 phosphatase positive colonies and remained trapped in a naïve pluripotent state, $\mathrm{RAS}^{\mathrm{KO}}$; $\mathrm{ERF}^{\mathrm{KO}}$

147 ESCs lost this ability (Fig. 2E). Our results demonstrate a predominant role for ERF controlling the 148 exit from naïve pluripotency.

\section{ERF controls the progression to primed pluripotency}

151 To obtain mechanistic insights on the rescue mediated by the loss of ERF during the exit from 152 naïve pluripotency, we performed RNA sequencing (RNAseq) analysis of ESC cultured under naïve 153 conditions (2iL) or differentiated into EpiLSC in FA. Principal component plot (PCA) analysis 154 segregated the samples based on their differentiation status alongside PC1, and MAPK activity 155 alongside PC2 (Fig. 3A). Interestingly, FA-RAS ${ }^{K O}$ ESC are localized at an intermediate state 156 between naïve and primed pluripotency (Fig. 3A). FA-RAS ${ }^{\mathrm{KO}}$ ESC are characterized by the 157 intermediate to high expression of naïve pluripotent markers as well as of primed associated 158 genes including OTX2 (Fig. 3B, C). Indeed, while naïve pluripotent ESC are characterized by a 159 NANOG $^{+} / \mathrm{OTX2}^{-} / \mathrm{OCT6}^{-}$state, FA-RAS ${ }^{\mathrm{KO}}$ ESC showed a NANOG ${ }^{+} / \mathrm{OTX2}^{+} / \mathrm{OCT6}^{-}$state (Fig. 3B, C). 160 Furthermore, although FA-RAS ${ }^{\text {lox/lox }}$ ESC have undetectable levels of ERF upon differentiation, FA161 RAS $^{\mathrm{KO}}$ ESC retain high ERF expression (Fig. $3 \mathrm{C}$ ). Using available datasets that evaluate temporal 162 transcriptional dynamics during the transition from naïve to primed pluripotency, we determined 163 that FA-RAS ${ }^{\mathrm{KO}}$ ESC transcriptionally resembled to ESC primed for 12-24 hours (Fig. 3D and fig. $164 \mathrm{~S} 3 \mathrm{~A})(18)$. Interestingly, FA-RAS ${ }^{\mathrm{KO}}$ ESC are reminiscent of the recently described intermediate 165 pluripotent states, rosette and formative pluripotent states (RSC, FSC and XPSC) (6-8). PCA analyses showed that PC1 (differentiation status) placed all intermediate pluripotent states in a

167 similar transcriptional space but PC2 segregated FA-RAS ${ }^{\text {KO }}$ ESC from rosette or formative 168 pluripotent states (Fig. 3E). Hierarchical clustering analysis based on the expression of naïve and 169 primed markers revealed that RSC and FA-RAS ${ }^{\mathrm{KO}}$ ESC are transcriptionally more comparable, 170 likely to the defective MAPK signaling in both intermediate states (Fig. 3F). Finally, we observed 171 that prolonged culture of FA-RAS ${ }^{K O}$ ESC in FGF2/Activin-A/XAV939 (FAX) conditions 172 demonstrated their trapping in this intermediate state of pluripotency (fig. S3B). Our results 173 showed that loss of ERF is necessary and sufficient to overcome the developmental blockage of 174 FA-RAS ${ }^{\mathrm{KO}}$ ESC in its intermediate pluripotent state. Indeed, RNAseq data revealed that loss of ERF 
175 in $\mathrm{RAS}^{\mathrm{KO}}$; $\mathrm{ERF}^{\mathrm{KO}} \mathrm{ESC}$ restored the overall gene expression profile to be indistinguishable from FA-

$176 \mathrm{RAS}^{\mathrm{lox} / \text { lox }}$. In summary, our data point to ERF as the MAPK-dependent switch that triggers full

177 commitment to primed pluripotency.

Chromatin-bound ERF ensures an optimal naïve pluripotency state

180 The expression of ERF correlates with high expression levels of naïve markers in vivo and in vitro 181 and is quickly downregulated upon induction to primed pluripotency (Fig. 1). Moreover, ERF is 182 enriched in a total of 2074 bona-fide ESC enhancers identified in RAS ${ }^{\text {KO }}$ ESC (Supplemental Data 183 S1 and 2) (9). Among these, ERF binds to most of the super-enhancers identified in ESC (198/231) 184 (Supplemental Data S3) $(9,22,23)$. ERF-bound super-enhancers are associated to highly 185 transcribed naïve genes (KLF4, ESRRB, PRDM14, NANOG, TBX3 or ZFP42) (some examples in fig. $186 \mathrm{~S} 4 \mathrm{~A}$ ) as well as to general pluripotent genes (OCT4 or SOX2). Although ERF is considered to be a 187 transcriptional repressor, we hypothesized that ERF might play a different role at ESC enhancers. 188 To explore the relevance of ERF at these enhancers, we first examined the level of occupancy of 189 the pluripotent transcription factors OCT4, SOX2 and NANOG (O, S and N, respectively) in the 190 subset of ERF-bound 2074 ESC enhancers. We observed increased occupancy of bound OSN at 191 these compared to a non-ERF bound randomized set of different 2074 enhancers (Fig. 4A). In 192 addition, ERF-bound ESC enhancers are also characterized by higher H3K27Ac levels, increased 193 p300 binding and chromatin accessibility detected by ATACseq (fig. S4B). These results suggested 194 that ERF could be regulating the activity of these enhancers, and thus, the expression of their 195 associated genes. Therefore, we examined the expression levels of essential naïve pluripotent 196 genes associated to ERF-bound super-enhancers in RAS lox/lox and ERF ${ }^{\text {KO }}$ ESC grown in 2iL 197 conditions. Interestingly, NANOG, PRDM14 and ZFP42 showed decreased expression in 2iL-ERF ${ }^{\text {KO }}$ 198 ESC, while TBX3, KLF4 and ESRRB do not (Fig. 4B). It has been shown that NANOG promotes 199 chromatin accessibility and binding of pluripotent factors to enhancers (24). Thus, we 200 hypothesized that reduced levels of NANOG could affect the expression of additional naïve201 associated genes. Indeed, ERF ${ }^{\mathrm{KO}}$ cells showed an overall decreased expression of the naïve 202 pluripotent transcriptional network (Fig. 4C). Consistent with these results, unidimensional PCA 203 analysis (PC1) segregating samples based on their differentiation status showed that 2iL-ERF 
204 ESC are biased toward differentiation (Fig. 4D). These combined results revealed that ESC

205 growing under naïve conditions required ERF binding at enhancers to maintain an optimal naïve

206 pluripotent state. To confirm this observation, we employed native Cut\&Run sequencing to 207 examine SOX2 and NANOG occupancy in RAS ${ }^{\text {lox/lox }}$ and ERF ${ }^{\text {KO }}$ ESC grown in 2iL conditions. As 208 predicted, both pluripotent transcription factors showed an overall decreased enrichment in ESC 209 enhancers in naïve ERF ${ }^{\mathrm{KO}}$ ESC (Fig. 4E, F and fig. S4C). These data support our findings and 210 revealed an unexpected unique role for ERF in promoting naïve pluripotency.

212 The naïve enhancer landscape is active in FA-RAS ${ }^{\mathrm{KO}}$ ESC

213 Inactivation of the naïve transcriptional network is necessary for the successful progression to 214 primed pluripotency. This is associated with decommission of naïve enhancers by 215 downregulation of naïve transcription factors including ESRRB, KLF4 or NANOG and a widespread 216 OCT4 genomic relocation (25). Indeed, OCT4 shifts from enhancers associated with key players 217 in naïve pluripotency and engages in new enhancer elements at genes implicated in post218 implantation development. Using datasets for OCT4 occupancy in ESC and EpiLSC and based on 219 the relative occupancy between both cellular states, we defined top OCT4 sites preferentially 220 enriched in ESC (4759 sites, OCT4 ${ }^{\text {ESC }}$ ), EpiLSC (2921 sites, OCT4 ${ }^{\text {EpiLSC}}$ ) and commonly shared

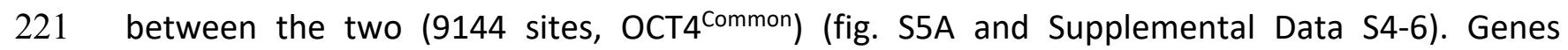
222 associated with OCT4 ${ }^{\mathrm{ESC}}$ sites are mostly downregulated during the transition to primed 223 pluripotency whereas genes associated to OCT4 ${ }^{\text {EpiLSC }}$ sites are upregulated (25). Out of all OCT4 224 bound sites, a total of 12.52\% (596/4759 ERF/OCT4 ${ }^{\text {ESC }}$ sites), 13.62\% (1246/9144 ERF/OCT4 Common 225 sites) and $0.15 \%$ (44/2921 ERF/OCT4 ${ }^{\text {EpiLSC }}$ sites) were co-occupied by ERF in naïve ESC (Fig. 5A).

226 This suggested that ERF does not play a specific role in genes associated with EpiLSC-specific 227 sequences but instead in regulating OCT4 ${ }^{\mathrm{ESC}}$ and OCT4 ${ }^{\text {Common }}$ sites. We first focused on OCT4 ${ }^{\text {ESC }}$ 228 sites as we observed that FA-RAS ${ }^{\mathrm{KO}}$ ESC remain trapped in an intermediate state between naïve 229 and primed pluripotency and showed expression of naïve pluripotent markers (Fig. 3A-C). 230 Differentially expressed genes associated with ERF/OCT4 ESC sites were mostly downregulated 231 during FA differentiation in RAS ${ }^{\text {lox/lox }}$ ESC (Fig. 5B). While many of these genes did not change 232 their expression level in FA-RAS ${ }^{\mathrm{KO}}$ ESC, those that changed showed a lower differential expression 
233 extent (Fig. 5B). Enhancer decommission of naïve enhancers by OCT4 relocation is followed by

234 changes in enhancer chromatin patterns including decreased levels of H3K27ac (24). Thus, we

235 examined whether the naïve enhancer landscape is still fully active in FA-RAS ${ }^{\mathrm{KO}}$ ESC by performing

236 native Cut\&Run sequencing to evaluate the levels of H3K27ac as a marker for active enhancers

237 and NANOG occupancy in ESC and EpiLSC from all genotypes. Interestingly, 2iL-RAS ${ }^{\mathrm{KO}}$ ESC showed

238 increased acetylation and NANOG occupancy at ERF/OCT4 ${ }^{\text {ESC }}$ sites (Fig. 5C, D), a phenotype that

239 is also observed in all OCT4 ${ }^{\text {ESC }}$ sites (fig. S5B). This increase in H3K27ac levels does not result in

240 major overall transcriptional changes and the expression level of naïve associated genes is similar

241 between RAS ${ }^{\text {lox/lox }}$ and RAS ${ }^{\text {KO }}$ ESC (Fig. 4C). This observation supports the idea that levels of

242 H3K27ac does not necessarily determine enhancer activity but rather discriminates between

243 active or poised enhancers (26). Exit from naïve pluripotency correlated with an overall decrease

244 in H3K27ac levels, negligible chromatin-bound NANOG and reduced expression of naïve-

245 associated genes in RAS ${ }^{\text {lox/lox }}$ ESC (Fig. 5C, D and fig. S5B). However, FA-RAS ${ }^{\text {KO }}$ ESC showed

246 elevated gene expression, NANOG occupancy and H3K27ac levels at ERF/OCT4 ${ }^{\text {ESC }}$ sites to, in some

247 cases, a comparable level as detected in naïve RAS lox/lox ESC (Fig. 5C, D). Together, these data

248 showed that naïve-specific associated OCT4 binding sites remain fully active in the FA-RAS ${ }^{\mathrm{KO}}$ ESC

249 intermediate state.

250 We next focused specifically on the regulatory sequences and expression level of naïve 251 transcription factors associated with ERF-bound super-enhancers. Among these, the expression

252 level of ERF-dependent genes (NANOG, PRDM14 and ZFP42) is unaffected in FA-RAS ${ }^{\mathrm{KO}}$ ESC and is 253 comparable to that of RAS ${ }^{\text {lox/lox }}$ ESCS as ERF is still expressed (Fig. 5E). However, ERF-independent 254 genes (ESRRB, TBX3 and KLF4) showed a significant decrease in expression, suggesting that 255 additional mechanisms ensure optimal expression of these genes in 2iL-ESC (Fig. 5E). Collectively, 256 these results demonstrated that FA-RAS ${ }^{\mathrm{KO}}$ ESC retain an active naïve transcriptional network. 257 Although some naïve markers showed a marked decreased expression at this stage, elevated 258 levels of NANOG, PRDM14 or ZFP42 likely sustain the naïve like-state in FA-RAS ${ }^{\mathrm{KO}}$ ESC mediated 259 by ERF-dependent mechanisms. 
262 OTX2 co-occupy binding sites with NANOG in FA-RAS ${ }^{\mathrm{KO}}$ ESC

263 Global reorganization of OCT4 genomic binding is driven by increased expression of OTX2 during 264 naïve to primed transition (25). In fact, ectopic OTX2 expression in 2iL-ESC shows that it behaves 265 as a pioneering factor engaging in previously inaccessible enhancer sites, relocates OCT4 to these 266 sites, and induces the expression of primed-associated genes (25). OTX2 expression is 267 independent of MEK signals but is efficiently repressed by the WNT pathway, thus explaining the 268 elevated levels of OTX2 in rosette-like ESC (6). High levels of OTX2 have also been found to be 269 associated to formative pluripotency $(7,8)$. In addition, we also detected similar high OTX2 levels 270 in FA-RAS ${ }^{K O}$ ESC compared to FA-RAS ${ }^{\text {lox/lox }}$ (Fig. 6A). We have shown that ERF binding sustains 271 optimal naïve transcription factor expression (Fig. 4) and thus, we asked whether ERF is involved 272 in OTX2 regulation. Indeed, ERF binds to the super-enhancer region associated with OTX2 in 273 RAS $^{\text {KO }}$ ESC. Furthermore, the low levels of OTX2 expression observed in 2iL-RAS ${ }^{\text {lox/lox }}$ ESC are 274 further decreased in 2iL-ERF ${ }^{\mathrm{KO}}$ ESC (Fig. 6A, B). This decrease correlates with lower NANOG and 275 SOX2 binding and suggests that ERF binding might prevent further OTX2 repression in the 276 absence of FGF signaling (Fig. 6B). In agreement, FA-RAS ${ }^{\text {KO }}$ ESC showed strong NANOG 277 enrichment as well as OTX2 itself, which could potentially sustain its own expression after 278 NANOG downregulation (fig. S6A). We next focused on the ERF/OCT4 ${ }^{\text {Common }}$ sites as they gained 279 OTX2 and H3K27ac enrichment in EpiLSC compared to ESC (24). Differentially expressed genes 280 associated with ERF/OCT4 ${ }^{\text {Common }}$ sites were mostly upregulated in FA-RAS ${ }^{\text {lox/lox }}$ (Fig. 6C). Similar 281 to what we observed with genes associated with ERF/OCT4 ${ }^{\mathrm{ESC}}$ sites, many of these genes did not 282 change their expression levels in FA-RAS ${ }^{K O}$ ESC and those that changed showed a lower 283 differential expression extent (Fig. 6C). Based on the elevated levels of NANOG expression in FA284 RAS $^{\mathrm{KO}}$ ESC, we hypothesized that ERF/OCT4 ${ }^{\text {Common }}$ sites also retained chromatin bound NANOG. 285 While FA-RAS ${ }^{\text {lox/lox }}$ showed negligible enrichment of NANOG at these sites, FA-RAS ${ }^{\text {KO }}$ ESC retained 286 NANOG bound (Fig. 6D). In addition, we also observed stronger OTX2 enrichment at these sites 287 in FA-RAS ${ }^{\mathrm{KO}}$ ESC compared to FA-RAS ${ }^{\text {lox/lox }}$ (Fig. 6D). Interestingly, we also detected OTX2 binding 288 in FA-RAS ${ }^{\mathrm{KO}}$ ESC at the ERF/OCT4 ${ }^{\mathrm{ESC}}$ sites, naïve associated sequences that are decommissioned 289 during the transition to EpiLSC (Fig. 6D). These observations were also confirmed globally in all 290 OCT4 sites (fig. S6B). Furthermore, OTX2 as well as NANOG were also strongly enriched at the 
291 OCT4 ${ }^{\text {EpiLSC }}$ sites in FA-RAS ${ }^{\text {KO }}$ ESC, suggesting that, besides OCT4, OTX2 might also relocate

292 additional naïve pluripotent factors (if expressed) to these sites prior to full differentiation (Fig.

293 6D and fig. S6B). Finally, we examined whether OTX2 and NANOG enrichment correlated with

294 increased expression of post-implantation epiblast genes associated with ERF/OCT4 ${ }^{\text {Common }}$ sites

295 in FA-RAS ${ }^{\mathrm{KO}}$ ESC. We found genes showing a similar level of expression in FA-RAS ${ }^{\mathrm{KO}}$ ESC compared

296 to FA-RAS ${ }^{\text {lox/lox }}$ (Fig. 6E and fig. S6C). Our data demonstrate that FA-RAS ${ }^{\text {KO }}$ ESC show promiscuous

297 OTX2 and NANOG binding at OCT4 ${ }^{\text {ESC }}$ sites and OCT4 ${ }^{\text {EpiLSC }}$ sites, which correlates with the

298 expression of both naïve and primed markers in preparation for the transition towards primed

299 pluripotency.

\section{ERF controls the expression of LIN28 proteins}

302 We next investigated how ERF controls the exit from the developmental blockage of RAS303 deficient cells by mining our RNAseq data (Fig. 3). By combining differential gene expression 304 between our different genetic conditions and differentiation status together with nearby ERF 305 binding occupancy, we identified LIN28A and B as putative regulators. Expression of LIN28A/B is 306 low in 2iL-ESC but is upregulated by FGF signaling during the transition to primed pluripotency. 307 Of note, LIN28A and/or LIN28B-deficient ESC showed impaired conversion into EpiLSC, revealing 308 a critical role for these proteins in regulating the exit from naïve pluripotency (27). Induction of 309 EpiLSC differentiation in RAS ${ }^{\text {lox/lox }}$ and ERF ${ }^{K O}$ ESC demonstrated efficient LIN28s upregulation 310 while FA-RAS ${ }^{K O}$ ESC showed low or negligible levels of LIN28A and LIN28B, respectively (Fig. 7A).

311 ERF deficiency restored the levels of LIN28 proteins in FA-RAS ${ }^{\mathrm{KO}}$; ERF ${ }^{\mathrm{KO}}$ (Fig. 7A). Interestingly,

312 2iL-ERF ${ }^{\mathrm{KO}}$ ESC showed already elevated levels of LIN28 proteins before differentiation, suggesting 313 that ERF mediates a direct negative regulation on these genes. Indeed, we found ERF bound to 314 an enhancer region in the second intron of LIN28 proteins in RAS ${ }^{K O}$ ESC (Fig. 7B and fig. S7A).

315 Moreover, transition to EpiLSC correlated with increased levels of H3K27ac at the LIN28 316 enhancers, and thus, LIN28 expression (Fig. 7B).

317 LIN28 proteins are RNA binding proteins known for binding to and inactivating the let-7 microRNA 318 family. Functionally, let-7 microRNAs target a number of mRNA transcripts for degradation 319 including MYC, RAS, HMGA2 and the two de novo methyltransferases DNMT3A and DNMT3B 
320 (28). Indeed, naïve ESC exhibit low levels of genome wide CpG methylation, which increase during

321 the transition to EpiLSC, correlating with the silencing of the naïve transcriptional program (29,

$32230)$. Accordingly, induction of RAS ${ }^{\text {lox/lox }}$ and ERF ${ }^{K O}$ ESC into EpiLSC by FA demonstrated efficient

323 upregulation of DNMT3 proteins while FA-RAS ${ }^{K O}$ ESC showed low levels of DNMT3B (Fig. 7A). As

324 expected, expression of DNMT3 proteins was efficiently rescued in FA-RAS ${ }^{\mathrm{KO}}$; ERF ${ }^{\mathrm{KO}}$ (Fig. 7A). We

325 next examined the levels of 5-methylcytosine $(5 \mathrm{mC})$ by dot blot in all different genotypes,

$326 \mathrm{RAS}^{\mathrm{lox} / \mathrm{lox}}, \mathrm{ERF}^{\mathrm{KO}}, \mathrm{RAS}^{\mathrm{KO}}$, and $\mathrm{RAS}^{\mathrm{KO}}$; $\mathrm{ERF}^{\mathrm{KO}}$, grown under naïve or primed conditions (fig. S7B). The

327 low levels of $5 \mathrm{mC}$ detected in 2iL rapidly increased upon FA treatment in RAS ${ }^{\text {lox/lox }}$, ERF ${ }^{\mathrm{KO}}$ and

$328 \mathrm{RAS}^{\mathrm{KO}}$; ERF ${ }^{\mathrm{KO}}$ ESC. However, FA-RAS ${ }^{\mathrm{KO}}$ ESC remained largely hypomethylated (fig. S7B). To get

329 further insights into methylation dynamics on a genome-wide manner, we performed reduced 330 representation bisulfite sequencing (RRBS) in all genotypes and pluripotent states (Fig. 7C, D)

331 (31). 2iL-RAS ${ }^{\text {lox/lox }}$ ESC presented low levels of methylation (6.55\% of analyzed CpG sites are 332 methylated), which were increased after differentiation (21.75\%). Furthermore, 2iL-ERF ${ }^{\mathrm{KO}}$ ESC 333 showed higher average methylation levels in 2iL (12.1\%) and FA (24.7\%) conditions, consistent 334 with their bias toward differentiation and their less optimal naïve transcriptional network (Fig. 335 7C, D). Conversely, RAS ${ }^{\mathrm{KO}}$ ESC showed extremely low methylation levels in $2 \mathrm{iL}$ conditions $(1.55 \%)$ 336 reaching similar levels to those found in 2iL-RAS ${ }^{\text {lox/lox }}$ ESC when differentiated (6.9\%) (Fig. 7C, D).

337 As expected, the defect in methylation observed in RAS ${ }^{\mathrm{KO}}$ ESC was rescued upon ERF deletion

338 (Fig. 7C, D). Interestingly, ERF ${ }^{\mathrm{KO}}$ ESC showed higher level of methylation around transcription start 339 sites as well as in CpG islands (Fig. 7E), especially after differentiation. Collectively, our data 340 showed that ERF regulates negatively the expression of Lin28 and DNMT3 proteins. The altered 341 expression of these proteins might underlie the global disbalance in the methylation levels at a 342 genome-wide level in ERF-deficient cells. 


\section{DISCUSSION}

350 Preceding implantation, the cells residing within the naïve ICM of the blastocyst transition 351 through a distinct phase of primed pluripotency in preparation for lineage commitment. This 352 transition is initiated by fibroblast growth factor 4 (FGF4)-dependent activation of the RAS/MAPK 353 pathway $(32,33)$. Despite the relevance of the MAPK pathway during this critical period, little is 354 known about how RAS proteins instruct this transition. Here, we used RAS-deficient ESC for a 355 careful dissection of the FGF pathway and demonstrated that the transcriptional factor ERF is the 356 sole MAPK-dependent switch that controls the naïve-to-primed transition.

357 FA-RAS ${ }^{\mathrm{KO}}$ ESC are characterized by an active naïve transcriptional network, high expression of 358 OTX2 and elevated expression of primed markers. This suggests that 1) activation of the primed transcriptional program does not require full exit from naïve pluripotency, and 2) FGF/MAPK is

360 the main pathway controlling the exit from naïve pluripotency. Indeed, our data revealed that 361 ERF imposes an exquisite level of coordination during the transition from naïve to primed 362 pluripotency. In the absence of MAPK signaling, chromatin bound ERF ensures elevated expression of naïve pluripotent factors, including NANOG (Fig. 4). High levels of NANOG maintain

364 the undifferentiated state of ESC in the absence of LIF and might shield naïve embryonic cells 365 from premature commitment $(24,34)$. Accordingly, ERF ${ }^{\mathrm{KO}}$ ESC showed decreased levels of 366 NANOG and undergo a partial exit from naïve pluripotency under 2iL conditions (Fig. 4). Thus, 367 our data suggest that ERF supports naïve pluripotency, at least partially, by sustaining elevated 368 levels of NANOG. ERF also controls negatively the expression of LIN28 proteins as $2 \mathrm{iL}^{\mathrm{L}-\mathrm{ERF}}{ }^{\mathrm{KO}} \mathrm{ESC}$ 369 showed increased levels of both LIN28A and B. Although LIN28 proteins regulate the expression 370 of DNMT3 proteins by sequestering let-7 microRNAs, we did not detect upregulation of the 371 methyltransferases in naïve ERF ${ }^{\mathrm{KO}}$ ESC (Fig. 7). This is likely due to high levels of PRDM14, which 372 represses expression of DNMT3 methyltransferases by recruiting Polycomb repressive complex 3732 to their promoters, and it is regulated by ERF (Fig. 5E) (35). Thus, coordinated PRDM14 374 downregulation and increased LIN28 expression through ERF-dependent mechanisms could 375 contribute to DNMT3 upregulation and increased methylation during the transition to primed 376 pluripotency. Although DNMT3A and DNMT3B are dispensable for a successful exit from naïve 377 pluripotency, de novo DNA methylation facilitates a timely progression towards a primed state 
378 (35). In fact, DNMT3A/B double knockout ESC exhibited a delayed exit from the naïve state and 379 defective expression of the post-implantation markers OCT6, FGF5 or OTX2 (36). This is ultimately 380 due to persistent NANOG expression after 2iL withdrawal as DNMT3A/B deficiency leads to 381 reduced CpG methylation at the proximal NANOG promoter $(36,37)$. Similarly, FA-RAS ${ }^{\mathrm{KO}}$ ESC 382 showed negligible expression of DNMT3B and, therefore, high levels of NANOG. Interestingly, 383 ERF $^{\mathrm{KO}}$ ESC showed increased DNA methylation compared to RAS ${ }^{\text {lox/lox }}$ ESC and is particularly 384 evident in genes and CpG islands after the transition to EpiLSC (Fig. 7E). Naïve ERF ${ }^{\mathrm{KO}}$ ESC have low 385 levels of DNMT3 expression but elevated levels of DNMT3L, the catalytically inactive regulatory 386 factor of de novo DNA methyltransferases (fig. S7C), which can contribute to this bias during 387 differentiation (38). Furthermore, DPPA3, also known as Stella and implicated in preventing 388 excessive DNA methylation by sequestering the E3 ubiquitin ligase UHRF1, is also decreased in $389 \operatorname{ERF}^{\mathrm{KO}} \operatorname{ESC}$ (Fig. S7C) $(39,40)$. Importantly, downregulation of DPPA3 mediated by DNMT3 390 methylation has been shown to be a key event in the naïve to primed conversion (41). 391 Collectively, elevated levels of DNMT3L and low levels of DPPA3 in naïve conditions could contribute to the aberrant level of methylation detected in FA-ERF ${ }^{K O}$. Nevertheless, these 393 changes in methylation do not result in major transcriptional changes in FA-ERF ${ }^{\mathrm{KO}}$ compared to 394 their respective control cells.

395 FA-RAS ${ }^{K O}$ ESC showed high expression of OTX2, which has been shown a critical transcription 396 factor for thew maintenance of intermediate states of pluripotency (7). The consequence of 397 having high levels of OTX2 in this context can be inferred by previous studies (25). Indeed, OTX2 398 over-expression has been shown to redirect OCT4 to previously inaccessible sites in EpiLSC, 399 decrease expression of naïve markers (TBX3 or ESRRB), and induce EpiLSC genes, such as FGF5 400 (25). Interestingly, these effects take place in the presence of MEKi, a situation that is mirrored 401 in FA-RAS ${ }^{\mathrm{KO}}$ ESC. The ability of OTX2 to engage new enhancer sequences not only depends on its 402 levels but also on the cooperative help of additional transcription factors (25). This cooperation 403 might explain the enrichment of OTX2 in active naïve enhancers in FA-RAS ${ }^{\mathrm{KO}}$ ESC. Moreover, our 404 data also showed that, in addition to OCT4, NANOG is mobilized to sites that become active in 405 EpiLSCs, which results in partial or full transcriptional activation (Fig. 6E). In this context, NANOG 406 relocation might facilitate OTX2 positioning as it has been shown to promote chromatin 
407 accessibility and TF recruitment together with BRG1, part of the large remodeling complex 408 SWI/SNF (24).

409 Finally, we showed that FA-RAS ${ }^{\mathrm{KO}}$ ESC can be maintained in culture under primed conditions and 410 could be a suitable genetic model to study intermediate states of pluripotency. Indeed, FA-RAS ${ }^{\text {KO }}$ 411 ESC mirrored transcriptionally cells that have departed 12-24 hours from naïve pluripotency and 412 are reminiscent of the recently described pluripotent intermediate states (6-8). Future studies

413 will be necessary to determine the similarity of all these intermediate states with corresponding 414 in vivo counterparts. Nevertheless, pluripotency can be considered as a dynamic property 415 associated to different transient stem cell states that can be recapitulated by using different 416 inhibitors and/or growth factors. By using RAS ${ }^{\mathrm{KO}}$ ESC we focused specifically on the FGF pathway 417 and the role of RAS proteins during the naïve to primed transition.

418 Future studies will be needed to determine if ERF plays a role in other cell fate decisions during 419 early embryonic development such as in primitive endoderm specification, where FGF4420 dependent activation of the MAPK pathway is also necessary. Moreover, the control of ERF over 421 PRDM14 expression suggested that ERF might also play an important role in germline fate (42).

422 In conclusion, here we demonstrated the essential role of ERF as regulator of the timely transition 423 to primed pluripotency. 
MATERIALS AND METHODS

\section{Embryo Culture}

All the animal work included here was performed in C57BL/6J mice obtained from the Jackson Laboratory in compliance with the NIH Animal Care \& Use Committee (ACUC) Guideline for

441 Breeding and Weaning. 4-weeks old female mice were injected intraperitoneally with 5IU

442 Pregnant Mare Serum Gonadotropin (PMSG, Prospec) followed by 5 IU human Chorionic 443 Gonadotropin (hCG, Sigma-Aldrich) 46-48 hours later. Alternatively, 8-weeks naturally pregnant 444 females were euthanized, and embryos collected in M2 media (MR-015-D, Sigma-Aldrich) at 445 indicated time points after hCG injection: E2.75, E3.5, E4.0, E4.75. The sex of embryos was not 446 determined. Embryos were fixed in 4\% Paraformaldehyde (Electron Microscopy Sciences) for 10 $447 \mathrm{~min}$, permeabilized for $30 \mathrm{~min}$ in $0.3 \%$ Triton X-100 and 0.1M Glycine in PBS $1 \mathrm{X}$ and blocked for 4481 hour (1\% BSA, 0.1\% Tween in PBS 1X). Embryos were incubated overnight with primary 449 antibodies (see STAR methods), washed in 0.1\% Tween in PBS $1 \mathrm{X}$ and incubated with the 450 secondary antibody accordingly for 1 hour at room temperature. Embryos were imaged using a 451 Nikon Ti2-E microscope (Nikon Instruments) equipped with a CSU-W1 spinning disk (Yokogawa), 452 Photometrics Prime BSI sCMOS (Photometrics), and 60x Nikon Apochromat TIRF objective (NA = 453 1.49). Z-stacks were acquired with a $x$-y pixel size of $0.11 \mathrm{~mm}$ and z-step of $0.9 \mathrm{~mm}$. For 454 quantification, embryo z-stack images were quantified using Imaris Bitplane (Oxford 455 Instruments). 3D surfaces were rendered based on nuclear DAPI-staining and the corresponding 456 regions were used to quantify the fluorescence intensity of ERF, NANOG, and KLF4. For 457 experiment shown in Fig. 1A shows one representative experiment with the following embryos 458 and cells used for quantification: Exp 1: E2.75: 3 Embryos, 24 cells in total. E3.5: 3 Embryos, 117 459 cells total, E4.0: 4 Embryos, 168 cells total, E4.75: 1 Embryo: 75 cells. Exp 2: E3.5: 8 embryos, 460 E3.75: 4 embryos, E4.0: 6 embryos.

\section{Cell culture and differentiation}

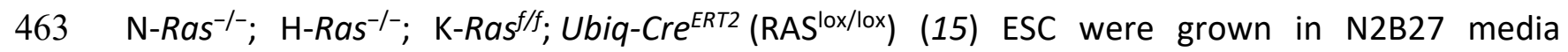
464 supplemented with 2i/LIF (1 mM PD0325901, 3 mM CHIR99021, both from Tocris and 1:500 LIF, 
465 made in house). N2B27 media consisted of a 1:1 mix of DMEM/F12 and Neurobasal Medium, 1X 466 N2 supplement, 1X B27 supplement, $0.1 \mathrm{mM}$ nonessential amino acids, $55 \mu \mathrm{M} \beta-$ 467 mercaptoethanol and 1\% penicillin/streptomycin (all from Life Technologies). Cells were 468 routinely cultured in $0.1 \%$ gelatinized plates and passaged with Accutase (Gibco) unless 469 otherwise indicated. To induce EpiLSC differentiation, cells were grown for few passages in plates 470 pretreated with $10 \mu \mathrm{g} / \mathrm{ml}$ polyL-ornithine and $5 \mu \mathrm{g} / \mathrm{ml}$ laminin (Corning). A total of 200,000471300,000 cells per $10 \mathrm{~cm}^{2}$ were plated on plates pretreated with $5 \mu \mathrm{g} / \mathrm{ml}$ Fibronectin (Millipore) 472 in N2B27 media supplemented with 1\% KOSR, $12 \mathrm{ng} / \mathrm{ml} \mathrm{FGF2} \mathrm{(R \& D} \mathrm{systems)} \mathrm{and} 20 \mathrm{ng} / \mathrm{ml}$ Activin 473 A (PeproTech) for 48 hours including daily media changes. For inducing CRE-mediated deletion 474 of K-RAS allele, we incubated RAS lox/lox ESC with $1 \mu \mathrm{M}$ 4-hydroxytamoxifen (4-OHT, Sigma-Aldrich) 475 for 6 days before performing any experiment. To maintain EpiLSC in culture, ESCs were plated in $476 \mathrm{~N} 2 \mathrm{~B} 27$ media with $12 \mathrm{ng} / \mathrm{ml}$ FGF2, $20 \mathrm{ng} / \mathrm{ml}$ Activin A and $1 \mathrm{mM}$ XAV939 on Fibronectin-coated 477 plates at a density of 10,000 cells per $\mathrm{cm}^{2}$. EpiLSC were passaged the first time with Accutase 478 including $1 \mathrm{mM}$ Y27632 to enhance plating efficiency. Media was changed every other day and 479 passaged every 2-3 days. HEK293T (American Type Culture Collection) cells were grown in 480 DMEM, 10\% FBS, and 1\% penicillin/streptomycin.

481 To target a short half-life form of eGFP (deGFP) in the endogenous REX1 gene, we generated a 482 targeting vector by inserting deGFP in $\mathrm{pCR}^{\circledR}$-Blunt II TOPO ${ }^{\circledR}$ (Zero Blunt TOPO PCR cloning kit, 483 Invitrogen). Homology arms PCR-amplified from endogenous sequences upstream of the start

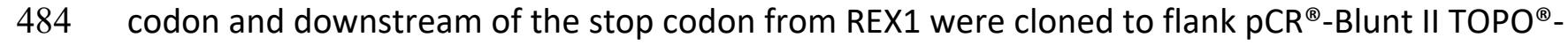
485 deGFP. In addition, specific small guide RNA (sgRNA) sequences targeting the surroundings of the 486 REX1 start codon were cloned into the plasmid pX330-U6-Chimeric_BB-CBh-hSpCas9 (Addgene, 487 42230) (44). The sequences of the sgRNAs were designed with the Genetic Perturbation Platform 488 sgRNA designer tool (https://portals.broadinstitute.org/gpp/public/analysis-tools/sgrna-design). 489 Both plasmids were transfected in ESC using Jetprime (Polyplus transfection) to induce the 490 targeting and cell sorted based on GFP intensity to isolate individual clonal ESC lines. See Table 491 S1 for primer information. 


\section{Clonogenicity Assay}

495 ESC were withdrawn of $2 \mathrm{i} /$ LIF for 48 hours and plated at single cell density $\left(50\right.$ cells/cm $\left.{ }^{2}\right)$ in N2B27

496 media with $2 \mathrm{i} /$ LIF on plates coated with $0.1 \%$ gelatin (Sigma). At day 5 , alkaline phosphatase

497 staining was performed using the Alkaline Phosphatase Detection Kit (Millipore). Colonies were

498 counted manually. At least three independent experiments with three replicates per experiment

499 were performed.

\section{Generation of self-organizing embryonic spheres}

502 ESC growing on gelatinized plates in N2B27 media with 2i/LIF were dissociated with Accutase and

503 washed with PBS before their resuspension in growth factor reduced Matrigel (Corning) at a 504 concentration of 10,000 cells $/ 20 \mu$ l of matrigel. The suspension was deposited in drops in 8 - $\mu$ well

505 Ibidi microplates and incubated at $37^{\circ} \mathrm{C}$ until the Matrigel solidified. Wells were then filled with

506 N2B27 media without 2i/LIF and cultured for $48-72$ hours at $37^{\circ} \mathrm{C}$ and $5 \% \mathrm{CO} 2$. ESC-derived 507 spheres were fixed in 4\% PBS-paraformaldehyde (PFA) for $10 \mathrm{~min}$ at room temperature. 508 Permeabilization was performed in PBS containing 0.3\% Triton X-100 (Sigma) and $0.1 \mathrm{M}$ glycine 509 (Sigma) for 30 minutes at room temperature. Spheres were incubated with primary antibodies 510 at $4{ }^{\circ} \mathrm{C}$ overnight, followed by incubation with corresponding fluorescently conjugated Alexa Fluor 511 secondary antibodies for 2 hours at room temperature. Both primary and secondary antibodies 512 were diluted in PBS containing 1\% BSA (Sigma) and 0.1\% Tween20 (Sigma). See Table S2 for 513 antibody information.

515 Western blot

516 Cells were lysed in $50 \mathrm{mM}$ Tris pH 8, $8 \mathrm{M}$ Urea (Sigma) and 1\% Chaps (Millipore) followed by $51730 \mathrm{~min}$ of shaking at $4^{\circ} \mathrm{C}$. $20 \mu \mathrm{g}$ of supernatants were run on $4 \%-12 \%$ NuPage Bis-Tris Gel 518 (Invitrogen) and transferred onto Nitrocellulose Blotting Membrane (GE Healthcare). 519 Membranes were blocked in 5\% skim milk (Millipore) and 0.1\% Tween 20 (Sigma) in PBS. 520 Membranes were incubated with the primary antibody overnight at $4^{\circ} \mathrm{C}$ (see STAR methods), 521 followed by incubation with HRP-conjugated secondary antibodies (1:5000) for $1 \mathrm{~h}$ at room 
522 temperature. Membranes were developed using SuperSignal West Pico PLUS (Thermo Scientific).

523 See Table S2 for antibody information.

\section{Dot blot}

526 Trypsinized ESC were lysed for $2-3$ hours at $55^{\circ} \mathrm{C}$ with lysis buffer $(100 \mathrm{mM}$ Tris- $\mathrm{HCl} \mathrm{pH} 8,5 \mathrm{mM}$ 527 EDTA, $0.2 \%$ SDS, $20 \mathrm{mM} \mathrm{NaCl}$ and $100 \mu \mathrm{g} / \mathrm{ml}$ Proteinase $\mathrm{K})$. DNA was isolated by adding an equal 528 volume of Phenol/Chloroform/Isoamyl to the samples and using phase-lock tubes (5PRIME Phase 529 Lock Gel ${ }^{\mathrm{TM}}$ Heavy, Quantabio), followed by an extraction with identical volume of Chloroform. 530 DNA was precipitated with 2 volumes of $100 \%$ Ethanol plus $0.3 \mathrm{M} \mathrm{NaAc}$, washed with $70 \%$ Ethanol 531 and resuspended in water. A total of $500 \mathrm{ng}$ of DNA was diluted in $0.3 \mathrm{M} \mathrm{NaOH}$ and denatured at $53242^{\circ} \mathrm{C}$ for 12 minutes. After incubation, the samples were rapidly transferred by spotting each 533 sample into a nitrocellulose membrane (Nitrocellulose Blotting Membrane, GE Healthcare). After 534 the transfer, DNA was crosslinked with a Stratalinker ${ }^{\circledR}$ UV crosslinker (Stratagene) using the 535 Autocrosslink setting. The membrane was blocked in 5\% skim milk (Millipore) and 0.1\% Tween 53620 (Sigma) in PBS, incubated with 1:500 dilution of the anti-5mC antibody (see STAR methods) 537 overnight at $4^{\circ} \mathrm{C}$, followed by incubation with HRP-conjugated secondary antibody (1:5000) for 1

$538 \mathrm{~h}$ at room temperature. Membrane was developed using SuperSignal West Pico PLUS (Thermo 539 Scientific). See Table S2 for antibody information.

\section{$541 \quad$ Flow cytometry}

542 For flow cytometry experiments, cells were dissociated into single cell suspensions and analyzed 543 for GFP gene expression using a FACS Fortessa (BD Biosciences). DAPI was added to detect cells 544 with compromised membrane integrity. Data was analyzed using FlowJo. At least two 545 independent experiments were performed.

\section{Immunofluorescence}

548 Cells were fixed in 4 \% Paraformaldehyde (Electron Microscopy Sciences) for 10 min at room 549 temperature, permeabilized in $100 \mathrm{mM}$ Tris- $\mathrm{HCl}$ pH 7.4, $50 \mathrm{mM}$ EDTA pH 8.0, 0.5\% Triton X-100 550 and incubated with the corresponding primary antibodies overnight (see STAR methods). This 
551 was followed by incubation with corresponding fluorescently conjugated Alexa Fluor secondary

552 antibodies for $2 \mathrm{~h}$ at room temperature. Both primary and secondary antibodies were diluted in

553 PBS containing 1\% BSA (Sigma) and 0.1\% Tween20 (Sigma). Images were acquired using either a

554 Nikon spinning disk confocal microscope (CSU-W1) or a Zeiss LSM880 Airyscan microscope. See

555 Table S2 for antibody information.

RNAseq and data analysis

558 RNA was isolated using the ISOLATE II RNA Mini Kit (Bioline) following manufacturer's 559 recommendations. DNA libraries for RNAseq analysis were prepared using NEBNext Ultra II

560 Directional RNA Library Prep Kit for Illumina (New England Biolabs, NEB) and NEBNext rRNA 561 Depletion Kit (Human/Mouse/Rat) (NEB) according to the manufacturer's protocol. Sequencing 562 was performed on the Illumina NextSeq550 (75bp pair-end reads). RNAseq reads were adapter 563 trimmed using fastp v.0.20.0 (45). Transcript expression was quantified via mapping to mouse 564 gencode v25 transcripts using salmon (46). Identification of differentially expressed genes 565 between samples was performed using DESEQ2 (PMID: 25516281). RNAseq coverage tracks 566 were generated by aligning RNAseq reads to UCSC version mm10 of the mouse genome using the 567 STAR v2.6.1a aligner (47) followed by application of the 'bamCoverage' utility from deeptools 568 (48) to generate signal track files with the following parameters: normalization=RPKM, 569 bin_size=50, smooth_length=1. For comparison to other published RNAseq data sets, gene 570 counts across samples were quantile-normalized using the limma package (49). Batch correction 571 was then performed on quantile-normalized counts using COMBAT (50). See Table S3 for further 572 software and algorithm information.

574 CUT\&RUN protocol

575 The CUT\&RUN protocol was slightly modified from $(51,52)$. In brief, cells were washed thrice 576 with Wash Buffer (20 mM HEPES-KOH pH 7.5, $150 \mathrm{mM} \mathrm{NaCl}, 0.5 \mathrm{mM}$ spermidine, Roche complete 577 Protease Inhibitor tablet EDTA free) and bound to activated Concanavalin A beads (Polysciences) 578 for 10 minutes at room temperature. Cells were then permeabilized in Digitonin Buffer $(0.05 \%$ 579 Digitonin and 0.1\% BSA in Wash Buffer) and incubated with corresponding antibodies at $4^{\circ} \mathrm{C}$ for 

antibody incubation, cells were washed with Digitonin Buffer and incubated with a hybrid protein A-protein G-Micrococcal nuclease (pAG-MNase) at $4^{\circ} \mathrm{C}$ for 1 hour. Samples were washed in Digitonin Buffer, resuspended in $150 \mu$ l Digitonin Buffer and equilibrated to $0^{\circ} \mathrm{C}$ on ice water for 5 minutes. To initiate MNase cleavage, $3 \mu \mathrm{l} 100 \mathrm{mM} \mathrm{CaCl}_{2}$ was added to cells and after 1 hour of digestion, reactions were stopped with the addition of $150 \mu \mathrm{l} 2 x$ Stop Buffer $(340 \mathrm{mM} \mathrm{NaCl}, 20$ mM EDTA, 4 mM EGTA, $0.02 \%$ Digitonin, $50 \mu \mathrm{g} / \mathrm{ml}$ RNase A, $50 \mu \mathrm{g} / \mathrm{ml}$ Glycogen). Samples were incubated at $37^{\circ} \mathrm{C}$ for 10 minutes to release DNA fragments and centrifuged at $16,000 \mathrm{~g}$ for

5885 minutes. Supernatants were collected and a mix of $1.5 \mu \mathrm{l} 20 \%$ SDS $/ 2.25 \mu \mathrm{l} 20 \mathrm{mg} / \mathrm{ml}$ Proteinase

$589 \mathrm{~K}$ was added to each sample and incubated at $65^{\circ} \mathrm{C}$ for 35 minutes. DNA was precipitated with ethanol and sodium acetate and pelleted by high-speed centrifugation at $4^{\circ} \mathrm{C}$, washed, air-dried and resuspended in $10 \mu 0.1 \times$ TE. See Table S2 for antibody information.

\section{Library preparation and sequencing}

594 The entire precipitated DNA obtained from CUT\&RUN was used to prepare Illumina compatible 595 sequencing libraries. In brief, end-repair was performed in $50 \mu$ of T4 ligase reaction buffer, $5960.4 \mathrm{mM}$ dNTPs, $3 \mathrm{U}$ of T4 DNA polymerase (NEB), $9 \mathrm{U}$ of T4 Polynucleotide Kinase (NEB) and $1 \mathrm{U}$ 597 of Klenow fragment (NEB) at $20^{\circ} \mathrm{C}$ for 30 minutes. End-repair reaction was cleaned using AMPure 598 XP beads (Beckman Coulter) and eluted in $16.5 \mu \mathrm{l}$ of Elution Buffer (10 mM Tris-HCl pH 8.5) 599 followed by A-tailing reaction in $20 \mu \mathrm{l}$ of dA-Tailing reaction buffer (NEB) with $2.5 \mathrm{U}$ of Klenow 600 fragment exo- (NEB) at $37^{\circ} \mathrm{C}$ for 30 minutes. The $20 \mu$ of the A-tailing reaction were mixed with 601 Quick Ligase buffer 2X (NEB), $3000 \mathrm{U}$ of Quick Ligase (NEB) and $10 \mathrm{nM}$ of annealed adaptor 602 (Illumina truncated adaptor) in a volume of $50 \mu \mathrm{l}$ and incubated at room temperature for $20 \mathrm{~min}$.

603 The adaptor was prepared by annealing the following HPLC-purified oligos: 5'604 Phos/GATCGGAAGAGCACACGTCT-3' and 5'-ACACTCTTTCCCTACACGACGCTCTTCCGATC*T-3'

605 (*phosphorothioate bond). Ligation was stopped by adding $50 \mathrm{mM}$ of EDTA, cleaned with AMPure 606 XP beads and eluted in $14 \mu \mathrm{l}$ of Elution Buffer. All volume was used for PCR amplification in a $60750 \mu \mathrm{l}$ reaction with $1 \mu \mathrm{M}$ primers TruSeq barcoded primer p7, 5'608 CAAGCAGAAGACGGCATACGAGATXXXXXXXXGTGACTGGAGTTCAGACGTGTGCTCTTCCGATC*T-3' 
and

TruSeq

barcoded

primer

p5

$5^{\prime}-$ AATGATACGGCGACCACCGAGATCTACACXXXXXXXXACACTCTTTCCCTACACGACGCTCTTCCGATC*T-

6113 3' (* represents a phosphothiorate bond and XXXXXXXX a barcode index sequence), and 2X Kapa

612 HiFi HotStart Ready mix (Kapa Biosciences). The temperature settings during the PCR 613 amplification were $45 \mathrm{~s}$ at $98^{\circ} \mathrm{C}$ followed by 15 cycles of $15 \mathrm{~s}$ at $98^{\circ} \mathrm{C}, 30 \mathrm{~s}$ at $63^{\circ} \mathrm{C}, 30 \mathrm{~s}$ at $72^{\circ} \mathrm{C}$

614 and a final 5 min extension at $72^{\circ} \mathrm{C}$. PCR reactions were cleaned with AMPure XP beads (Beckman

615 Coulter), run on a $2 \%$ agarose gel and a band of $300 \mathrm{bp}$ approximately was cut and gel purified

616 using QIAquick Gel Extraction Kit (QIAGEN). Library concentration was determined with KAPA

617 Library Quantification Kit for Illumina Platforms (Kapa Biosystems). Sequencing was performed

618 on the Illumina NextSeq550 (75bp pair-end reads).

\section{Cut\&Run data processing}

621 Data were processed using a modified version of Cut\&RunTools (53). Reads were adapter 622 trimmed using fastp v.0.20.0 (45). An additional trimming step was performed to remove up to $6236 \mathrm{bp}$ adapter from each read. Next, reads were aligned to the mm10 genome using bowtie2 (54) 624 with the 'dovetail' and 'sensitive' settings enabled. macs2 (55) was used to call peaks with q625 value cutoff $<0.01$. Normalized (RPKM) signal tracks were generated using the 'bamCoverage' 626 utility from deepTools with parameters bin-size $=25$, smooth length $=75$, and 'center_reads' and 627 'extend_reads' options enabled (48). See Table S3 for further software and algorithm 628 information.

\section{Processing for published ChIP datasets}

631 External next generation sequencing data were downloaded from the Sequence Read Archive 632 (SRA) and analyzed as follows. These analyses include a re-analysis of our original datasets (9). 633 Reads were aligned to the mm10 genome using bowtie2 (54). Duplicate reads were removed 634 using MarkDuplicates from the Picard toolkit ("Picard Toolkit." 2019. Broad Institute, GitHub 635 Repository. http://broadinstitute.github.io/picard/). Normalized (RPKM) signal tracks were 636 generated bamCoverage utility from deepTools (48), using the parameters bin-size=25, smooth 637 length=75, 'center_reads' and 'extend_reads'. For paired-end data, read mates were extended 
638 to the fragment size defined by the two read mates. For single-end ChIP-seq data, reads were

639 extended to the estimated fragment length estimated by phantompeakqualtools (56). See Table

640 S3 for further software and algorithm information.

641

642 Identification of OCT4 binding sites in ESC and EpiLSC

643 Fastq files from published OCT4 ChIP-seq data for ESC (SRR1202455, SRR1202456), EpiLSC plus 644 and minus Activin-A (SRR1202468, SRR1202469), and associated input controls (SRR1202465, SRR1202464, SRR1202477 SRR1202478) (25) were downloaded from the Sequence Read Archive

646 (SRA). Single-end reads were aligned to the mm10 genome using bwa (57). Duplicate reads were 647 removed using MarkDuplicates from the Picard toolkit (“Picard Toolkit.” 2019. Broad Institute, 648 GitHub Repository. http://broadinstitute.github.io/picard/), and peaks for each sample were 649 called using macs2 (55) with q-value cutoff < 0.01 and extension length determined using 650 phantompeakqualtools (56). Diffbind (58) using the DeSeq2 method was used to determine 651 differentially bound peaks, treating EpiLSC plus and minus Activin-A samples as replicate 652 experiments as was done in the original study (25). Peaks were determined to be ESC- or EpiLC653 specific if they differed by 2 -fold read concentration with $p$-val<0.01 and FDR $<0.03$. A subset of 654 peaks with $>$ mean read concentration for EpiLC and ESC with $<0.5$ fold difference were selected 655 as "common" or shared peaks. See Table S3 for further software and algorithm information.

\section{RRBS data processing}

658 DNA libraries for RRBS analysis were prepared using the Premium RRBS kit (Diagenode) following 659 manufacturer's recommendations. Sequencing was performed on the Illumina NextSeq550 660 (75bp single-end reads). Single-end RRBS reads were adapter and quality trimmed (phred33 661 score $>=20$ ) using trimgalore $\quad$ v0.6.5 662 (http://www.bioinformatics.babraham.ac.uk/projects/trim_galore) with the RRBS option 663 invoked. Bismark v0.22.1 (59) was used to align reads to UCSC version mm10 of the mouse 664 genome. CpG methylation was extracted using bismark ignoring the first 4 bases of the read after 665 inspection of $\mathrm{m}$-bias plots. Differentially methylated regions were called using the methylKit 666 package (60). See Table S3 for further software and algorithm information. 


\section{QUANTIFICATION AND STATISTICAL ANALYSIS.}

669 All information regarding statistical details of the experiments, number of experiments, statistical

670 tests used, number of cells/experiments can be found in the corresponding figure legends o

671 methods section.

672

673

674

675

676

677

678

679

680

681

682

683

684

685

686

687

688

689

690

691

692

693

694

695

696 


\section{REFERENCES}

1. J. Wray, T. Kalkan, A.G. Smith, The ground state of pluripotency. Biochem. Soc. Trans. 38, 1027-32 (2010).

2. J. Nichols, A. Smith, A. Naive and primed pluripotent states. Cell Stem Cell 4, 487-492 (2009).

3. L. Weinberger, M. Ayyash, N. Novershtern, J.H. Hanna, Dynamic stem cell states: naive to primed pluripotency in rodents and humans. Nat. Rev. Mol. Cell Biol. 17, 155-69 (2016).

4. Q.L. Ying, J. Wray, J. Nichols, L. Batlle-Morera, B. Doble, J. Woodgett, P. Cohen, A. Smith, The ground state of embryonic stem cell self-renewal. Nature 453, 519-23 (2008).

5. P.J. Tesar, J.G. Chenoweth, F.A. Brook, T.J. Davies, E.P. Evans, D.L. Mack, R.L., Gardner, R.D. McKay, New cell lines from mouse epiblast share defining features with human embryonic stem cells. Nature 448, 196-199 (2007).

6. A. Neagu, E. van Genderen, I. Escudero, L. Verwegen, D. Kurek, J. Lehmann, J. Stel, R.A.M.

8. L. Yu, Y. Wei, H. Sun, A.K. Mahdi, C.P. Arteaga, M. Sakurai, D.A. Schmitz, C. Zheng, E.D.

9. C. Mayor-Ruiz, T. Olbrich, M. Drosten, E. Lecona, M. Vega-Sendino, S. Ortega, O. Dominguez, M. Barbacid, S. Ruiz O. Fernández-Capetillo, ERF deletion rescues RAS deficiency in mouse embryonic stem cells. Genes\&Dev 32, 568-576 (2018).

10. D.N. Sgouras, M.A. Athanasiou, G.J. Jr. Beal, R.J. Fisher, D.G. Blair, G.J. Mavrothalassitis, ERF: an ETS domain protein with strong transcriptional repressor activity, can suppress ets- 
associated tumorigenesis and is regulated by phosphorylation during cell cycle and mitogenic stimulation. EMBO J. 14, 4781-4793 (1995).

11. L. Le Gallic, D. Sgouras, G. Jr. Beal, G. Mavrothalassitis, Transcriptional repressor ERF is a Ras/mitogen-activated protein kinase target that regulates cellular proliferation. Mol. Cell. Biol. 19, 4121-4133 (1999).

12. L. Le Gallic, L., Virgilio, P. Cohen, B. Biteau, G. Mavrothalassitis, ERF nuclear shuttling, a continuous monitor of Erk activity that links it to cell cycle progression. Mol. Cell. Biol. 24, 1206-18 (2004).

13. E. Vorgia, A. Zaragkoulias, I. Peraki, G. Mavrothalassitis, Suppression of Fgf2 by ETS2 repressor factor (ERF) is required for chorionic trophoblast differentiation. Mol. Reprod. Dev. 84, 286-295 (2017).

14. S. Nowotschin, M. Setty, Y.Y. Kuo, V. Liu, V., Garg, R. Sharma, C.S. Simon, N. Saiz, R. Gardner, S.C. Boutet, D.M. Church, P.A. Hoodless, A.K. Hadjantonakis, D. Pe'er, The emergent landscape of the mouse gut endoderm at single-cell resolution. Nature 569, 361-367 (2019).

15. M. Drosten, A. Dhawahir, E.Y. Sum, J. Urosevic, C.G. Lechuga, L.M. Esteban, E. Castellano, C. Guerra, E. Santos, M. Barbacid, Genetic analysis of Ras signalling pathways in cell proliferation, migration and survival. EMBO J. 29, 1091-1104 (2010).

16. K. Hayashi, H. Ohta, K. Kurimoto, S. Aramaki, M. Saitou Reconstitution of the mouse germ cell specification pathway in culture by pluripotent stem cells. Cell 146, 519-532 (2011).

17. F. Nakaki, K. Hayashi, H. Ohta, K. Kurimoto, Y. Yabuta, M. Saitou, Induction of mouse germcell fate by transcription factors in vitro. Nature 501, 222-226. (2013).

18. P. Yang, S.J. Humphrey, S. Cinghu, R. Pathania, A.J. Oldfield, D. Kumar, D. Perera, J.Y.H. Yang, D.E. James, M. Mann, R. Jothi, Multi-omic Profiling Reveals Dynamics of the Phased Progression of Pluripotency. Cell Syst. 8, 427-445 (2019).

19. I. Bedzhov, M. Zernicka-Goetz, Self-organizing properties of mouse pluripotent cells initiate morphogenesis upon implantation. Cell 156, 1032-1044 (2014).

20. M.N. Shahbazi, A. Scialdone, N. Skorupska, A. Weberling, G. Recher, M. Zhu, A. Jedrusik, L.G. Devito, L. Noli, I.C. Macaulay, C. Buecker, Y. Khalaf, D. Ilic, T. Voet, J.C. Marioni, M. Zernicka- 
Goetz Pluripotent state transitions coordinate morphogenesis in mouse and human embryos. Nature 552: 239-243 (2017).

21. T. Kalkan, N. Olova, M. Roode, C. Mulas, H.J. Lee, I. Nett, H. Marks, R. Walker, H.G. Stunnenberg, K.S. Lilley, J. Nichols, W. Reik, P. Bertone, A. Smith, Tracking the embryonic stem cell transition from ground state pluripotency. Development 144, 1221-1234 (2017).

22. D. Hnisz, B.J. Abraham, T.I. Lee, A. Lau, V. Saint-André, A.A. Sigova, H.A. Hoke, R.A. Young, Super-enhancers in the control of cell identity and disease. Cell 155, 934-947 (2013).

23. W.A. Whyte, D.A. Orlando, D. Hnisz, B.J. Abraham, C.Y. Lin, M.H. Kagey, P.B. Rahl, T.I. Lee, R.A. Young, Master transcription factors and mediator establish super-enhancers at key cell identity genes. Cell 153, 307-319 (2013).

24. V. Heurtier, N. Owens, I. Gonzalez, F. Mueller, C. Proux, D. Mornico, P. Clerc, A. Dubois, P. Navarro, The molecular logic of Nanog-induced self-renewal in mouse embryonic stem cells. Nat. Commun. 10, 1109 (2019).

26. T. Zhang, Z. Zhang, Q. Dong, J. Xiong, B. Zhu, Histone H3K27 acetylation is dispensable for

27. J. Zhang, S.Ratanasirintrawoot, S. Chandrasekaran, Z. Wu, S.B. Ficarro, C. Yu, C.A. Ross, D. Cacchiarelli, Q. Xia, M. Seligson, G. Shinoda, W. Xie, P. Cahan, L. Wang, S.C. Ng. S. Tintara, C. Trapnell, C. Onder, Y.H. Loh, T. Mikkelsen, P. Sliz, M.A. Teitell, J.M. Asara, J.A. Marto, H. Li,

28. J. Balzeau, M.R. Menezes, S. Cao, J.P. Hagan, The LIN28/let-7 Pathway in Cancer. Front. Genet. 8, 31 (2017).

29. G. Auclair, S. Guibert, A. Bender, M. Weber, Ontogeny of CpG island methylation and specificity of DNMT3 methyltransferases during embryonic development in the mouse. Genome Biol. 15, 545 (2014). 
30. S. Takahashi, S., Kobayashi, I. Hiratani Epigenetic differences between naïve and primed pluripotent stem cells. Cell Mol. Life Sci. 75, 1191-1203 (2018)

31. A. Meissner, A., Gnirke, G.W. Bell, B. Ramsahoye, E.S. Lander, R. Jaenisch, Reduced representation bisulfite sequencing for comparative high-resolution DNA methylation analysis. Nucleic Acids Res. 33, 5868-5877 (2005).

32. M. Kang, A. Piliszek, J. Artus, A.K. Hadjantonakis, FGF4 is required for lineage restriction and salt-and-pepper distribution of primitive endoderm factors but not their initial expression in the mouse. Development 140: 267-279 (2013).

33. Y. Yamanaka, F. Lanner, J. Rossant, FGF signal-dependent segregation of primitive endoderm and epiblast in the mouse blastocyst. Development 137: 715-724 (2010).

34. K. Mitsui, Y. Tokuzawa, H. Itoh, K. Segawa, M. Murakami, K. Takahashi, M. Maruyama, M., Maeda, S. Yamanaka, The homeoprotein Nanog is required for maintenance of pluripotency

35. M. Yamaji, J. Ueda, K. Hayashi, H. Ohta, Y. Yabuta, K. Kurimoto, R. Nakato, Y. Yamada, K. Shirahige, M. Saitou, PRDM14 ensures naive pluripotency through dual regulation of signaling and epigenetic pathways in mouse embryonic stem cells. Cell Stem Cell 12, 368-382 (2013).

36. M.A. Li, P.P. Amaral, P. Cheung, J.H. Bergmann, M. Kinoshita, T. Kalkan, M. Ralser, S. Robson, F. von Meyenn, M. Paramor, F. Yang, C. Chen, J. Nichols, D.L. Spector, T. Kouzarides, L. He, A. Smith, A IncRNA fine tunes the dynamics of a cell state transition involving Lin28, let-7 and de novo DNA methylation. Elife 6, e23468 (2017).

37. C.R. Farthing, G. Ficz, R.K. Ng, C.F. Chan, S. Andrews, W. Dean, M. Hemberger, W. Reik, Global mapping of DNA methylation in mouse promoters reveals epigenetic reprogramming of pluripotency genes. PLoS Genet. 4, e1000116 (2008).

38. N. Veland, Y. Lu, S. Hardikar, S., Gaddis, Y. Zeng, B. Liu, M.R. Estecio, Y. Takata, K. Lin, M.W. Tomida, J. Shen, D. Saha, H. Gowher, H., Zhao, T. Chen, DNMT3L facilitates DNA methylation partly by maintaining DNMT3A stability in mouse embryonic stem cells. Nucleic Acids Res. 47, 152-167. (2019). 
39. W. Du, Q. Dong, Z. Zhang, B. Liu, T. Zhou, R.M. Xu, H. Wang, B. Zhu, Y. Li, Stella protein facilitates DNA demethylation by disrupting the chromatin association of the RING fingertype E3 ubiquitin ligase UHRF1. J Biol Chem. 294, 8907-8917 (2019).

40. T. Nakamura, Y. Arai, H. Umehara, M. Masuhara, T. Kimura, H. Taniguchi, T. Sekimoto, M. Ikawa, Y. Yoneda, M. Okabe, S. Tanaka, K. Shiota, T. Nakano, PGC7/Stella protects against

47. A. Dobin, C.A. Davis, F. Schlesinger, J. Drenkow, C. Zaleski, S. Jha, P. Batut, M. Chaisson, T.R. Gingeras, STAR: ultrafast universal RNA-seq aligner. Bioinformatics 29: 15-21. (2013).

41. H. Sang, D. Wang, S. Zhao, J. Zhang, Y. Zhang, J. Xu, X. Chen, Y. Nie, K. Zhang, S. Zhang, Y. Wang, N. Wang, F. Ma, L. Shuai, Z. Li, N. Liu, Dppa3 is critical for Lin28a-regulated ES cells naïve-primed state conversion. J. Mol. Cell Biol. 11, 474-488 (2019).

42. N. Grabole, J. Tischler, J.A. Hackett, S. Kim, F. Tang, H.G. Leitch, E. Magnúsdóttir, M.A. Surani, Prdm14 promotes germline fate and naive pluripotency by repressing FGF signaling and DNA methylation. EMBO Rep. 14, 629-637. (2013).

43. C. Galonska, M.J. Ziller, R. Karnik, A. Meissner, Ground State Conditions Induce Rapid Reorganization of Core Pluripotency Factor Binding before Global Epigenetic Reprogramming. Cell Stem Cell 17, 462-470 (2015).

44. L. Cong, F.A. Ran, D. Cox, S. Lin, R. Barretto, N. Habib, P.D. Hsu, X. Wu, W. Jiang, L.A. Marraffini, F. Zhang, Multiplex genome engineering using CRISPR/Cas systems. Science, 339: 819-23 (2013)

45. S. Chen, Y. Zhou, Y., Chen, J. Gu, fastp: an ultra-fast all-in-one FASTQ preprocessor. Bioinformatics 34: 884-i890 (2018)

46. R. Patro, G. Duggal, M.I. Love, R.A. Irizarry, C. Kingsford, Salmon provides fast and bias-aware quantification of transcript expression. Nat. Methods 14, 417-419 (2017).

48. F. Ramírez, D.P. Ryan, B. Grüning, V. Bhardwaj, F. Kilpert, A.S. Richter, S. Heyne, F. Dündar, T. Manke, deepTools2: a next generation web server for deep-sequencing data analysis. Nucleic Acids Res 44: 160-165 (2016) 
49. M.E. Ritchie, B. Phipson, D. Wu, Y. Hu, C.W. Law, W. Shi, G.K. Smyth, limma powers differential expression analyses for RNA-sequencing and microarray studies. Nucleic Acids Res. 43: e47 (2015).

50. W.E. Johnson, C. Li, A. Rabinovic, Adjusting batch effects in microarray expression data using empirical Bayes methods. Biostatistics 8: 118-127 (2007).

51. P.J. Skene, S. Henikoff, An efficient targeted nuclease strategy for high-resolution mapping of DNA binding sites. Elife 6, e21856 (2017).

52. M.P. Meers, T.D. Bryson, J.G. Henikoff, S. Henikoff, Improved CUT\&RUN chromatin profiling tools. Elife 8, e46314 (2019).

53. Q. Zhu, N. Liu, S.H. Orkin, G.C. Yuan, CUT\&RUNTools: a flexible pipeline for CUT\&RUN processing and footprint analysis. Genome Biol. 20: 192 (2019).

54. B. Langmead, S.L. Salzberg, Fast gapped-read alignment with Bowtie 2. Nat. Methods 9: 357359. (2012).

55. Y. Zhang, T. Liu, C.A. Meyer, J. Eeckhoute, D.S. Johnson, B.E. Bernstein, C. Nusbaum, R.M.Myers, M. Brown, W. Li, X.S. Liu, Model-based analysis of ChIP-Seq (MACS). Genome Biol. 9: R137. (2008).

56. P.V. Kharchenko, M.Y. Tolstorukov, P.J. Park, Design and analysis of ChIP-seq experiments for DNA-binding proteins. Nat. Biotechnol. 26: 1351-1359 (2008).

57. H. Li, R. Durbin, Fast and accurate short read alignment with Burrows-Wheeler transform. Bioinformatics 25, 1754-1760 (2009).

58. R. Stark G.D. Brown DiffBind: Differential Binding Analysis of ChIP-Seq Peak Data. Bioconductor. Available online at: http://bioconductor.org/packages/release/bioc/html/DiffBind.html. (2011).

59. F. Krueger, S.R. Andrews, Bismark: a flexible aligner and methylation caller for Bisulfite-Seq applications. Bioinformatics 27: 1571-1572 (2011).

60. A. Akalin, M. Kormaksson, S. Li, F.E. Garrett-Bakelman, M.E. Figueroa, A. Melnick, C.E. Mason, methylKit: a comprehensive $\mathrm{R}$ package for the analysis of genome-wide DNA methylation profiles. Genome Biol. 13, R87 (2012). 
61. D. van Dijk, R. Sharma, J. Nainys, K. Yim, P. Kathail, A.J. Carr, C. Burdziak, K.R. Moon, C.L. Recovering Gene Interactions from Single-Cell Data Using Data Diffusion. Cell 174: 716729.e27 (2018).

62. J. Wu, B. Huang, H. Chen, Q. Yin, Y. Liu, Y. Xiang, B. Zhang, B., Liu, Q. Wang, W. Xia, W. Li, Y. Li, J. Ma, X. Peng, H. Zheng, J. Ming, W. Zhang, J. Zhang, G. Tian, F. Xu, Z. Chang, J. Na, X. Yang, W. Xie, The landscape of accessible chromatin in mammalian preimplantation embryos. Nature 534, 652-657 (2016).

873 
894 Acknowledgments: We thank Bechara Saykali for critical reading of the manuscript, Christa

895 Buecker for sharing reagents, Mariano Barbacid for sharing and Sagrario Ortega for isolating 896 RAS ${ }^{\text {lox/lox }}$ ESC. David Goldstein and the CCR Genomics Core for sequencing support and Ferenc 897 Livak and the CCR Flow cytometry Core for experimental support. Research in S.R. laboratory is 898 supported by the Intramural Research Program of the NIH. T.O. is supported by a Helen Hay 899 Whitney fellowship. Author contributions: M.V-S. and S.R. conceived the study. M.V-S., T.O., 900 designed and performed experiments. C.N.D. and M.F. provided technical support. D.T. and P.C.F. 901 analyzed sequencing data. A.D.T. and M.J.K. analyzed confocal microscopy data. S.R. supervised 902 the study and wrote the manuscript with comments from all authors. Competing interests: The 903 authors declare no competing interests. Data and materials availability: All data needed to 904 evaluate the conclusions in the paper are present in the paper and/or the Supplementary 905 Materials. The RNA-seq and Cut\&Run data generated in this study have been deposited in the 906 GEO database under accession GSE162581. Additional data related to this paper may be 907 requested from the authors. 

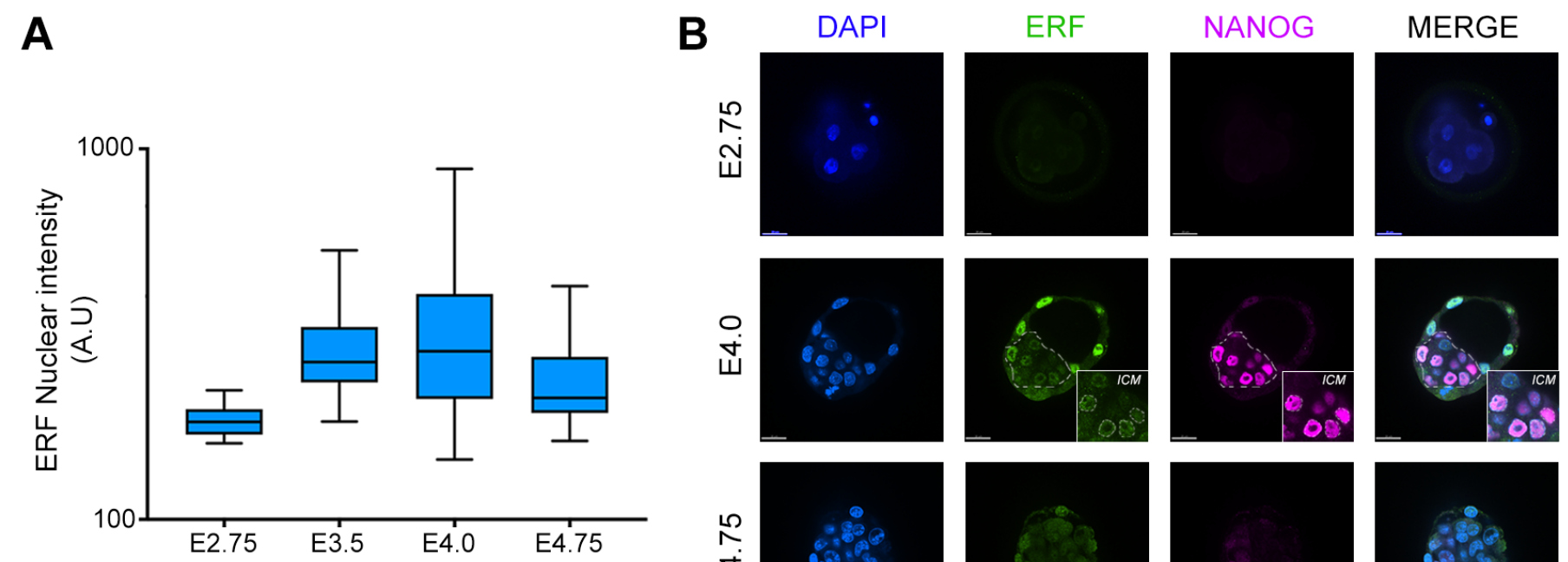

$\operatorname{MERGE}_{(\max \text { proj.) }}$
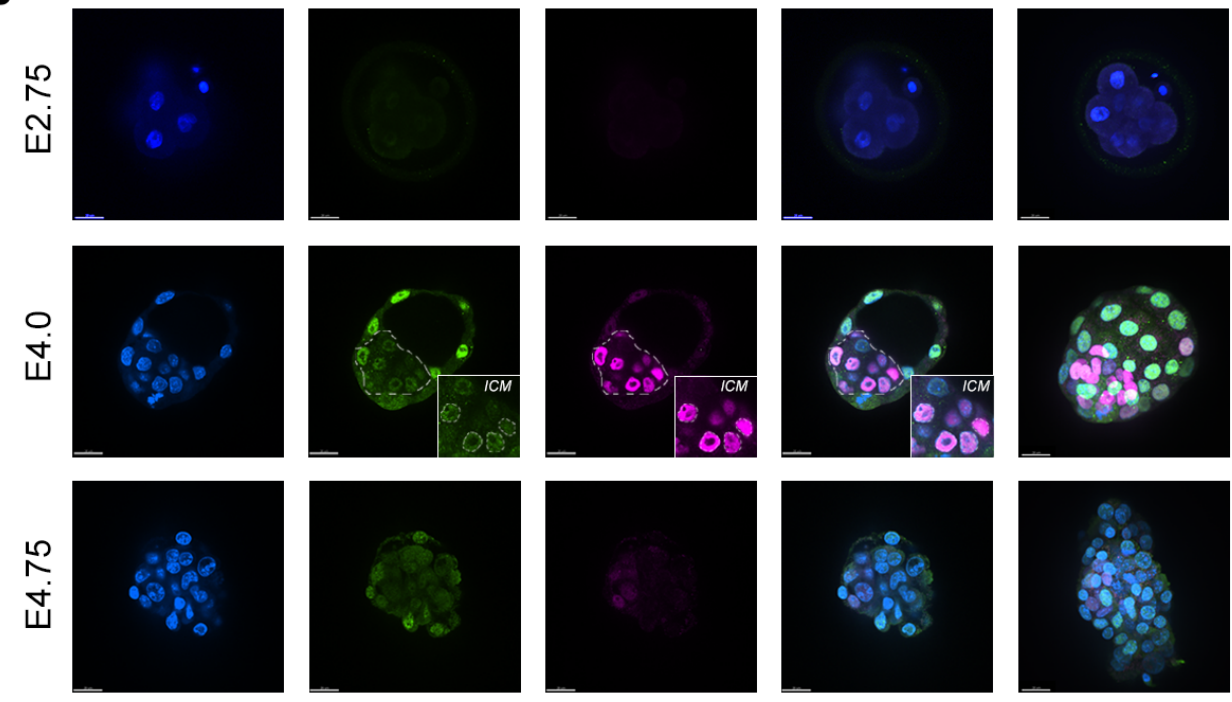

C

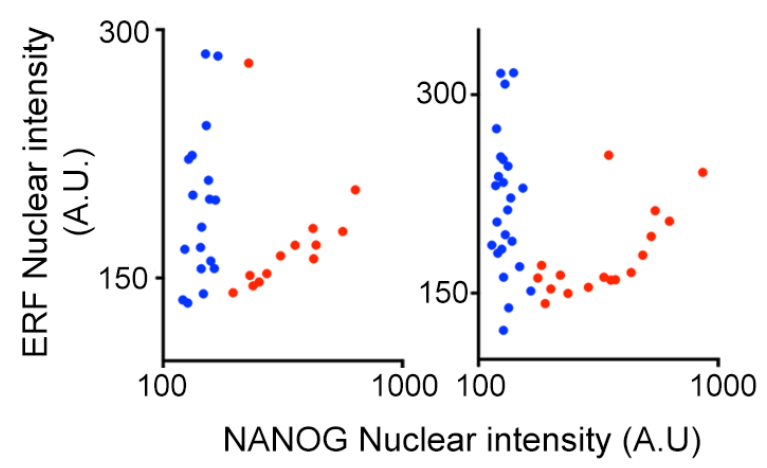

D
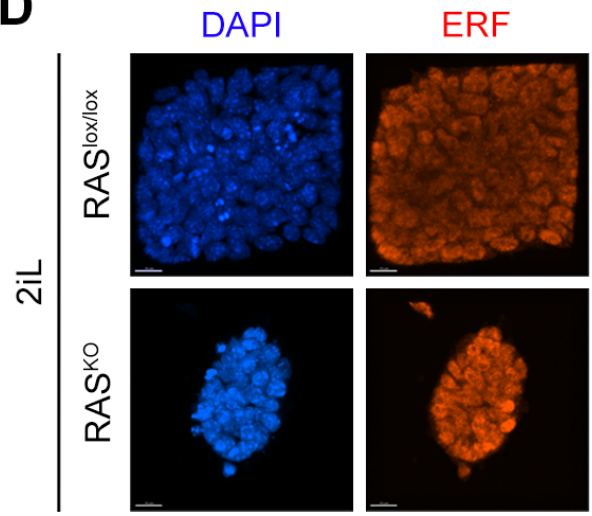

NANOG
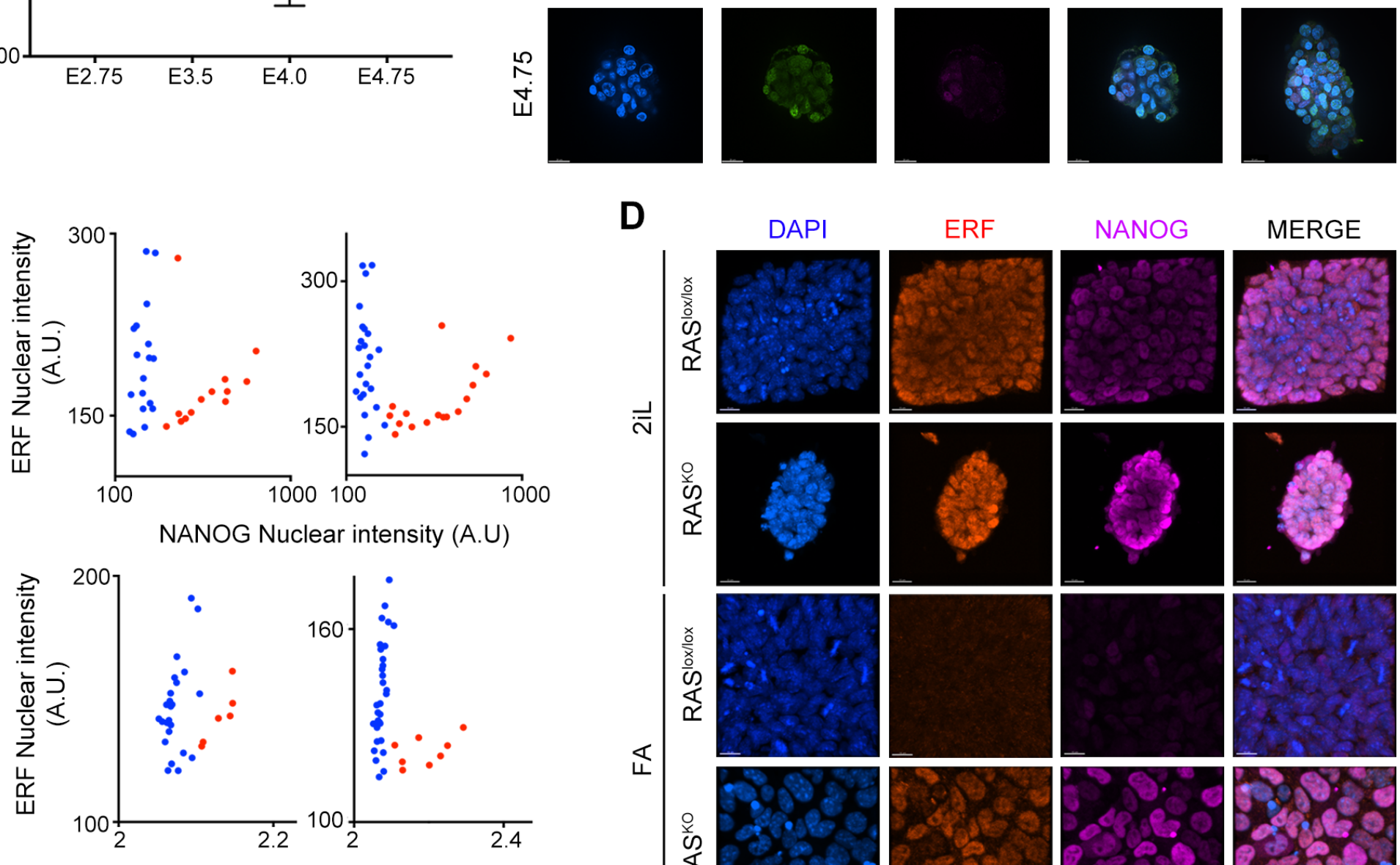

KLF4 Nuclear intensity (A.U)

- Trophectoderm $\bullet$ ICM
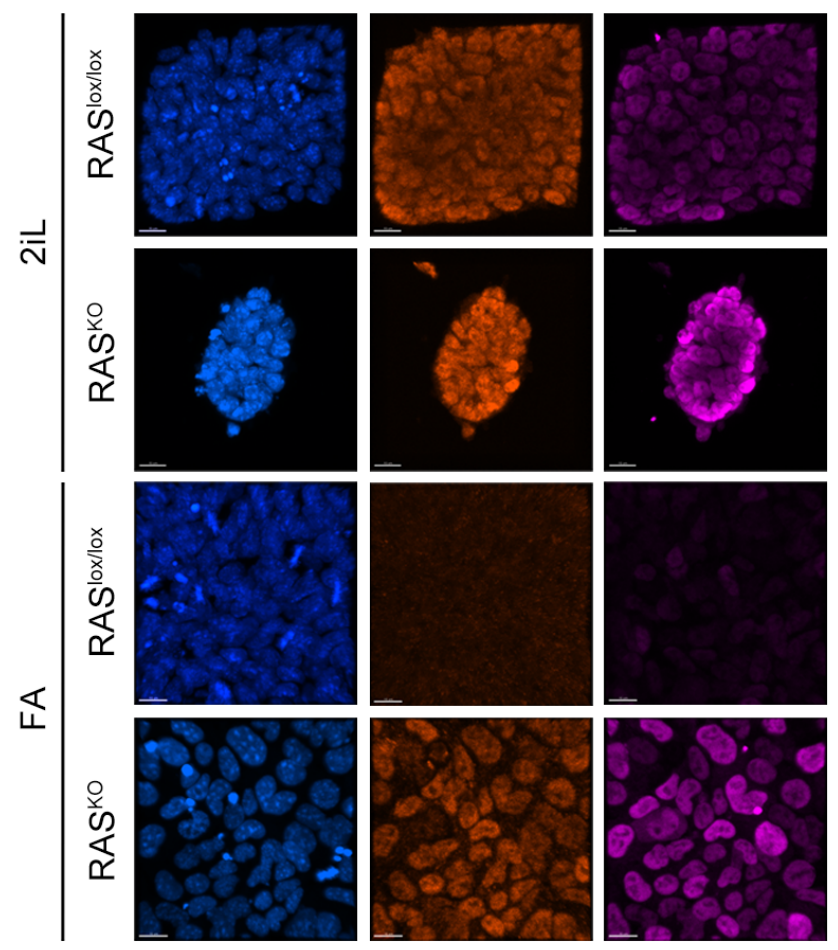

MERGE
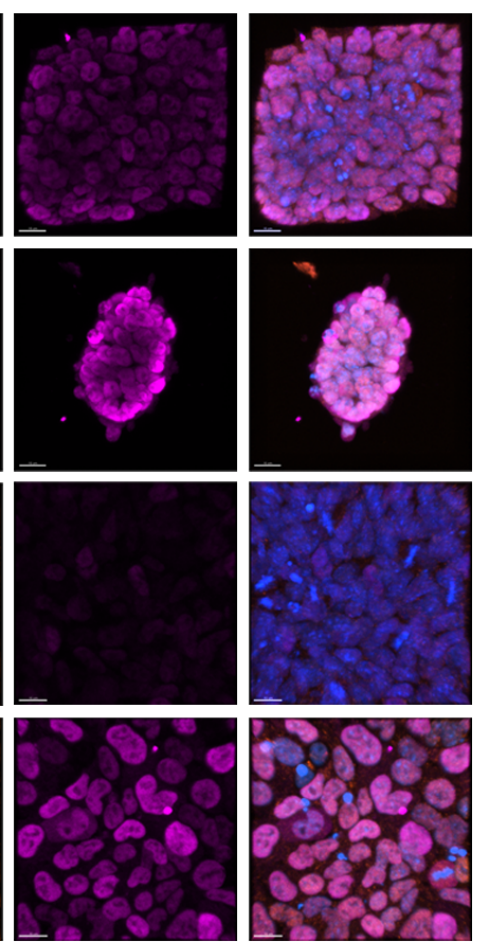

Figure 1 
923 Fig. 1. ERF expression correlates with naïve pluripotency markers. (A) Graph showing mean

924 nuclear fluorescent intensity for ERF in mouse embryos at different days of embryonic

925 development (E). (B) Immunofluorescence analysis of NANOG and ERF in mouse embryos at

$926 \mathrm{E} 2.75, \mathrm{E} 4.0$ and E4.75. Note that ERF is expressed in both ICM and TE. However, at E4.75 ERF and

927 NANOG are mostly downregulated in epiblast cells. Dashed line highlights the ICM. DAPI was

928 used to visualize nuclei. Scale bars, $20 \mu \mathrm{m}$. (C) Graphs showing relative nuclear fluorescence

929 intensity of ERF and NANOG (upper plots) and ERF and KLF4 (lower plots). Every dot represents

930 one single nucleus, and each plot corresponds to an individual E3.5 embryo. Two representative

931 examples are shown but at least 10 embryos were analyzed. (D) Immunofluorescence analysis of

932 2iL and FA treated RAS ${ }^{\text {lox/lox }}$ and RAS ${ }^{K O}$ ESC and stained for ERF (red) and NANOG (purple). DAPI

933 was used to visualize nuclei. Scale bars, $15 \mu \mathrm{m}$. 
A
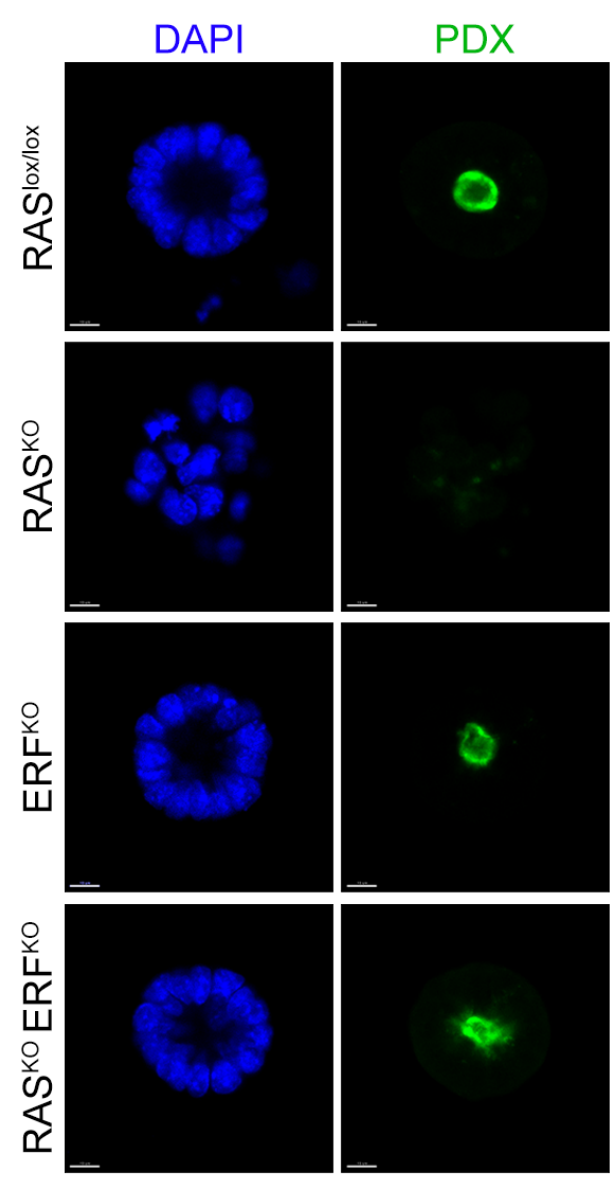

D

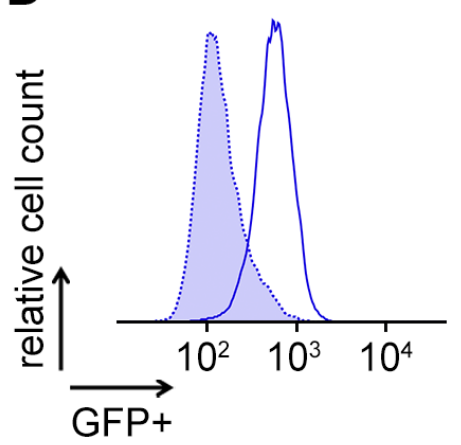

B

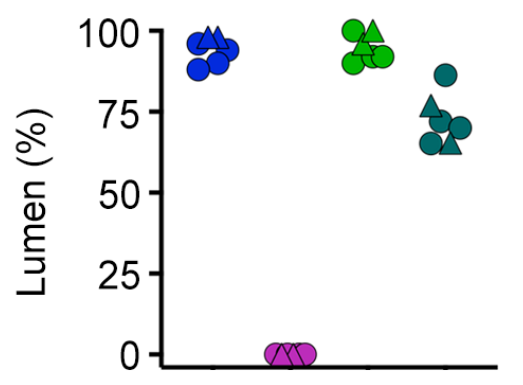

- RAS ${ }^{10 x / 10 x}$

- RAS ${ }^{K O}$

O ERFKO

- RAS ${ }^{K O}$ ERFKO $^{\mathrm{K} O}$

- Exp. 1

$\Delta$ Exp. 2
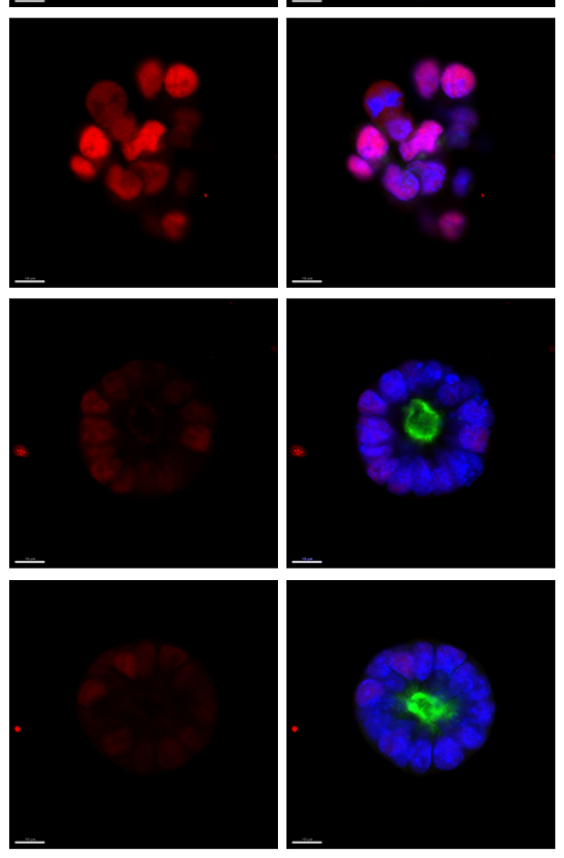

E

C

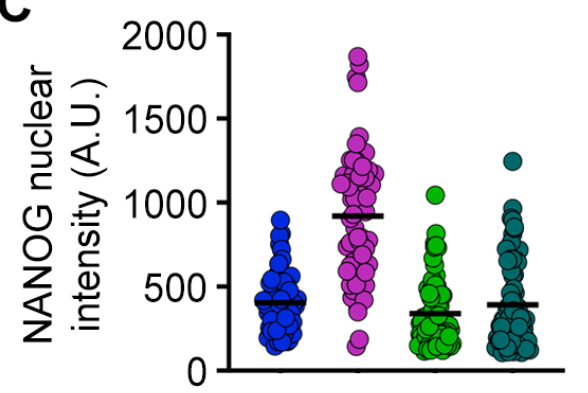

- RAS $S^{\operatorname{lox} / 0 x}$

- RAS ${ }^{\mathrm{KO}}$

- ERFKO

- RAS $^{\mathrm{KO}} \mathrm{ERF}^{\mathrm{KO}}$

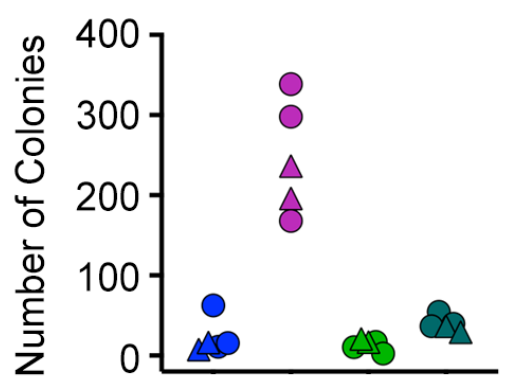

- RAS $S^{\operatorname{lox} / 0 x}$

- RASKO

- ERFKO

- RAS $^{\mathrm{KO}}$ ERF $^{\mathrm{KO}}$

- Exp. 1

$\Delta$ Exp. 2

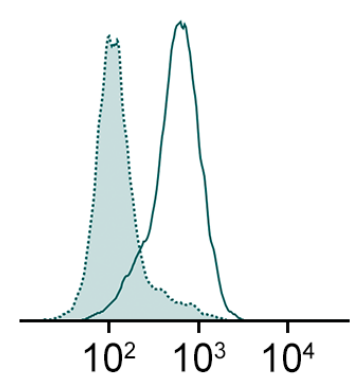

$\square$ RAS $^{\text {lox/lox }}$

$\square$ RAS $^{\mathrm{KO}}$

$\square$ ERF $^{\mathrm{KO}}$

$\square$ RAS $^{K O}$ ERF $^{K O}$

$\square$ 2iL

$\square$ FA 
952 Fig. 2. Successful exit from naïve pluripotency requires downregulation of ERF. (A) Central

953 confocal optical sections of RAS ${ }^{\text {lox/lox }}, E^{2} F^{K O}, R^{2} S^{K O}$, and RAS ${ }^{K O}$; ERF ${ }^{K O}$ embryonic cell rosettes

954 embedded in matrigel 48 hours after seeding and stained for NANOG (red) and podocalyxin (PDX;

955 green). DAPI was used to visualize nuclei. Scale bars, $10 \mu \mathrm{m}$. (B) Graph showing the percentage of

956 embryonic rosettes generating a lumen (PDX+) in all genotypes. Two independent experiments

957 are shown and at least, a total of 50 rosettes were counted per sample. (C) Graph showing mean

958 NANOG nuclear fluorescence intensity per nuclei in embryonic rosettes 48 hours after seeding.

959 One representative experiment is shown and a total of 70 nuclei from different rosettes were

960 counted per sample. (D) Flow cytometry analysis of REX1-deGFP reporter ESC from all genotypes

961 in 2iL-ESC and EpiLSC after 48 hours of induction with FA. Three independent experiments were

962 performed but one representative experiment is shown. (E) Graph showing the number of

963 alkaline phosphatase positive colonies in a colony forming assay using ESC from all genotypes.

964 Two independent experiments are shown with at least two technical replicates. 
A

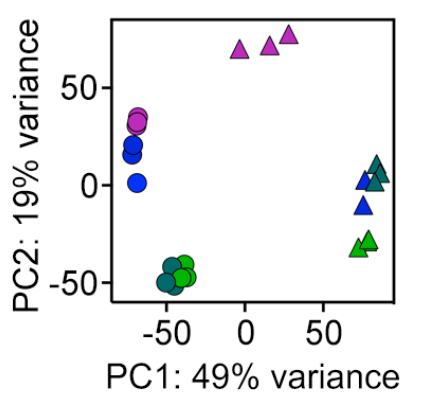

RAS lox/lox

RASKO

O ERFKO

RAS ${ }^{K O}$ ERF $^{K O}$

B
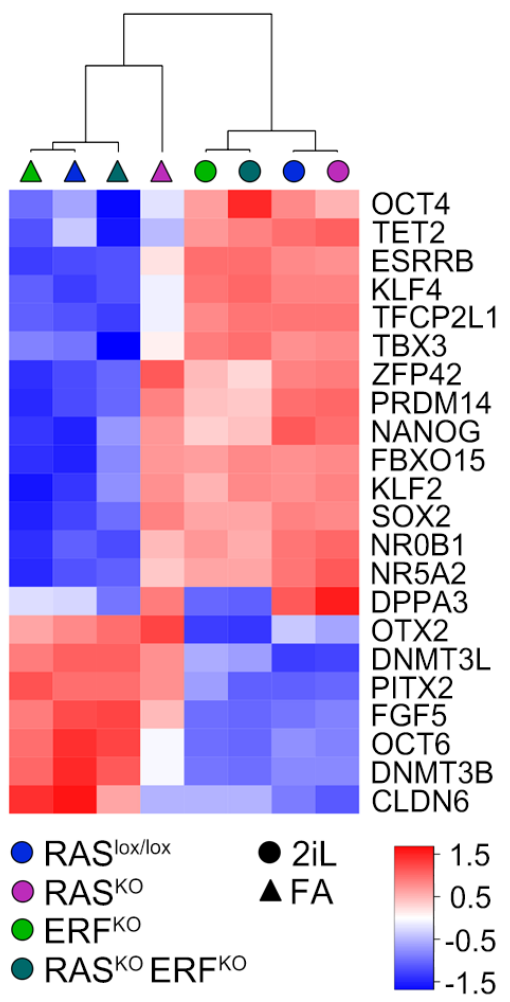

$\mathbf{E}$

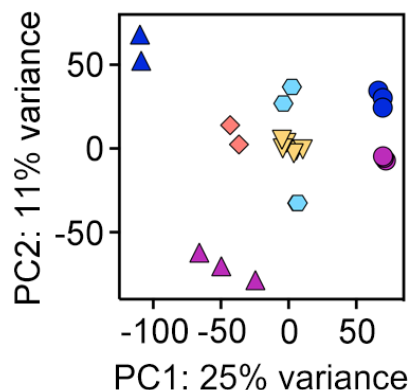

C

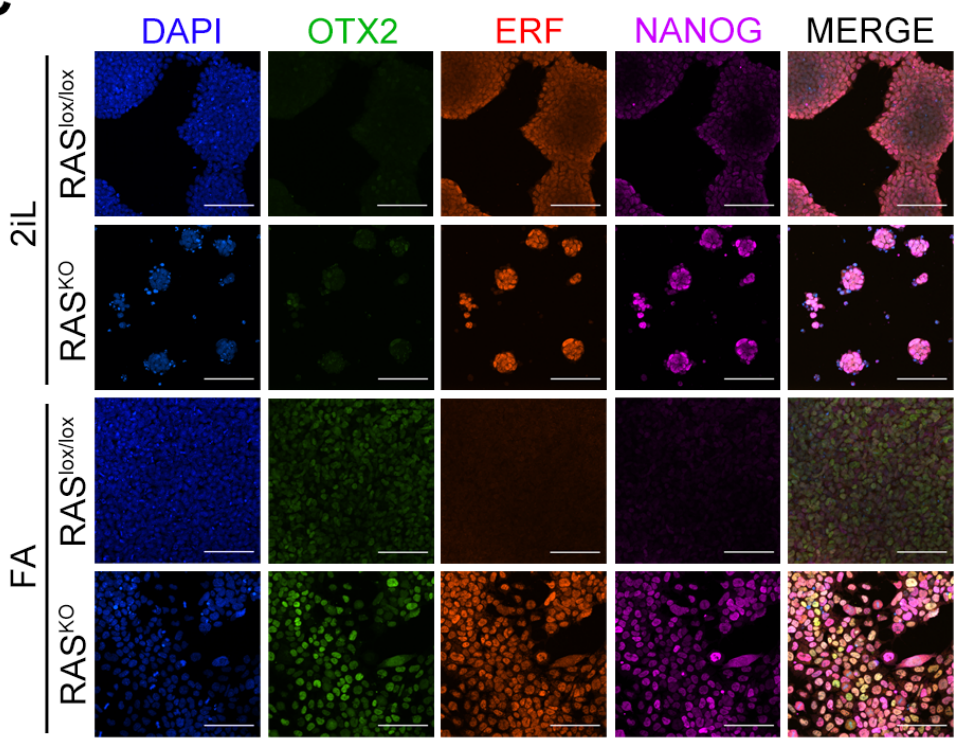

D

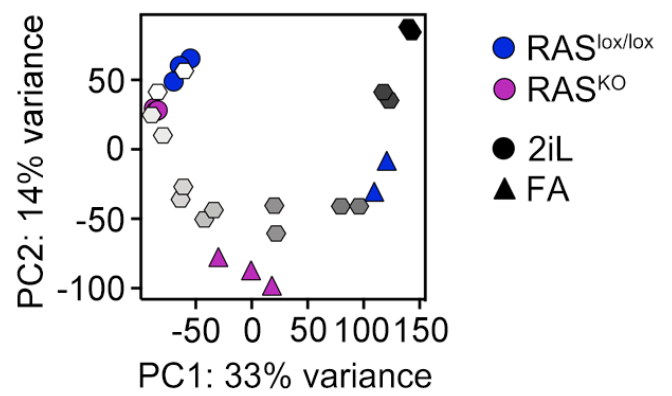

- 2iL

- FA $1 \mathrm{~h}$

- FA 6h

- FA $12 \mathrm{~h}$

- FA 24h

- FA 36h

- FA 48h

- FA 72h
F

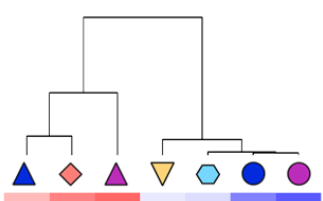

OTX2

DNMT3L

FGF5

PITX2

CLDN6

OCT6

DNMT3B

TET2

FBXO15

ZFP42

NANOG

PRDM14

KLF2

SOX2

NR5A2

NROB1

ESRRB

KLF4

DPPA3

TBX3

TFCP2L1

OCT4
RAS ${ }^{\text {lox/lox }}$

- RAS ${ }^{\mathrm{KO}}$

- 2iL

$\triangle \mathrm{FA}$

$\diamond \mathrm{RSC}$

$\checkmark F S C$

$\nabla$ XPSC

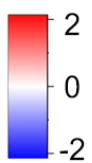


981 Fig. 3. ERF controls the transition to primed pluripotency. (A) PCA plot of RNAseq data of $982 \mathrm{RAS}^{\mathrm{lox} / \mathrm{lox}}, \mathrm{ERF}^{\mathrm{KO}}, \mathrm{RAS}^{\mathrm{KO}}$, and RAS ${ }^{\mathrm{KO}}$; $\mathrm{ERF}^{\mathrm{KO}} \mathrm{ESC}$ cultured in naïve conditions (2iL) or induced to 983 differentiate to EpiLSC (FA) for 48 hours. Three replicates per condition are shown. (B) Heatmap 984 generated from RNAseq data from samples described in A) showing the average from three 985 replicates. (C) Immunofluorescence analysis of 2iL and FA treated RAS ${ }^{\text {lox/lox }}$ and RAS ${ }^{\mathrm{KO}}$ ESC stained 986 for OTX2 (green), ERF (red) and NANOG (purple). DAPI was used to visualize nuclei. Scale bars, $987100 \mu \mathrm{m}$. (D) PCA plot of RNAseq datasets (three replicates) showing 2iL and FA treated RAS 988 and RAS ${ }^{\mathrm{KO}}$ ESC along with RNAseq datasets (two replicates) from a time course experiment during 989 EpilSC induction (0, 1, 6, 12, 24, 36, 48 and 72 hours) (18). (E) PCA plot of RNAseq datasets 990 showing 2iL and FA treated RAS ${ }^{\text {lox/lox }}$ and RAS ${ }^{\mathrm{KO}}$ ESC along with RNAseq datasets from RSC (ESC 991 lines grown in LIM (LIF, IWP2 (WNT inhibitor) and MEKi), FSC and XPSC (6-8). At least two 992 replicates are shown. (F) Heatmap generated from RNAseq data from samples described in (E) 993 showing the average from two or three replicates as applicable to each sample. 
A
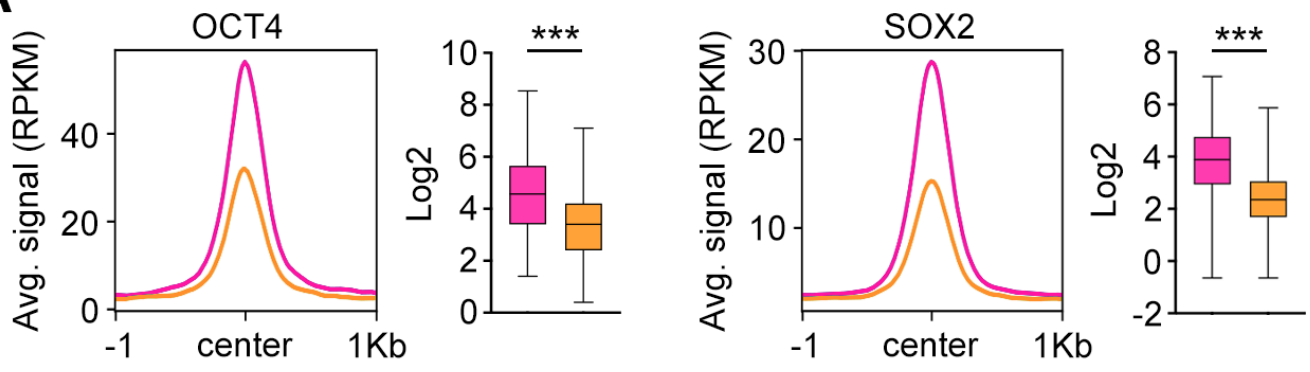

— ERF sites (Enhancers) — Random sites (Enhancers)

B

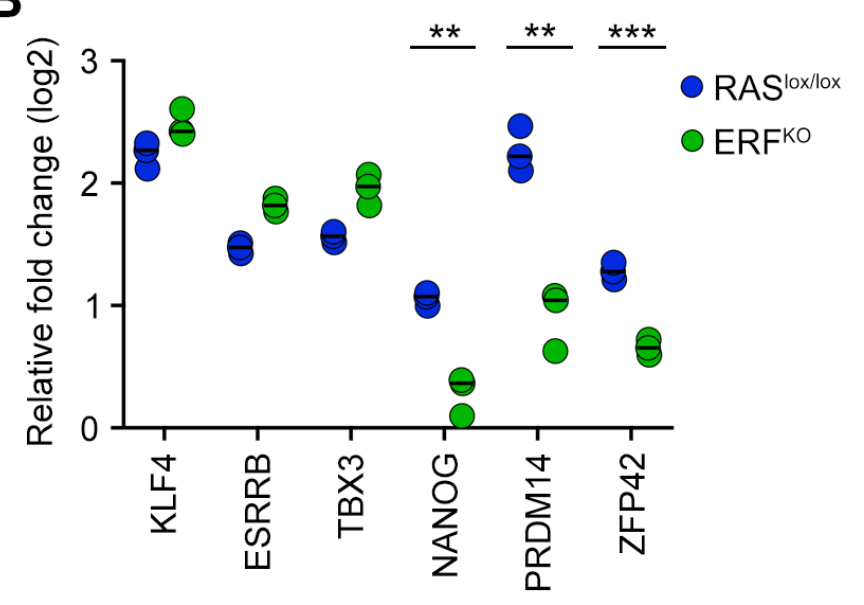

$\mathbf{E}$

ERF sites (Enhancers)
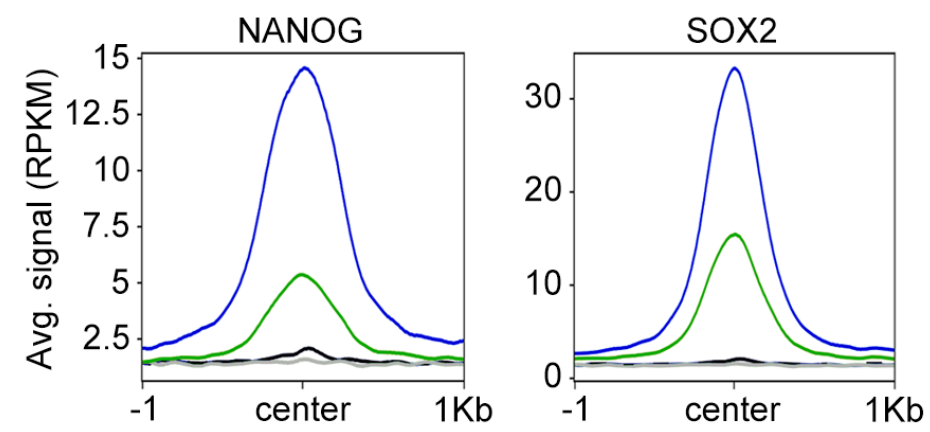

- $\operatorname{RAS}^{\operatorname{lox} / \operatorname{lox}}(\lg G)$

$-\operatorname{ERF}^{\mathrm{KO}}(\lg \mathrm{G})$

- RAS ${ }^{10 \times 10 x}$

- ERF'o
C

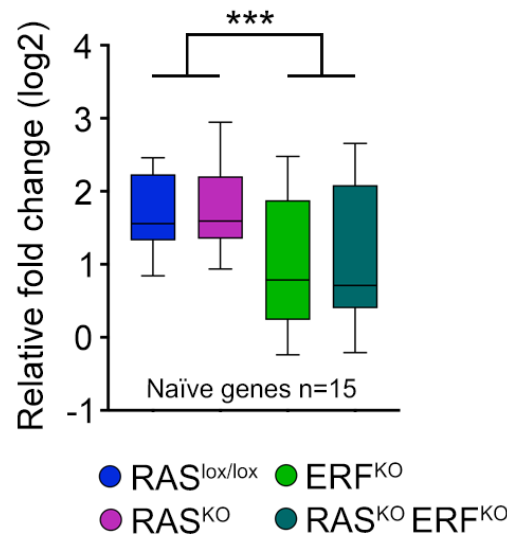

F

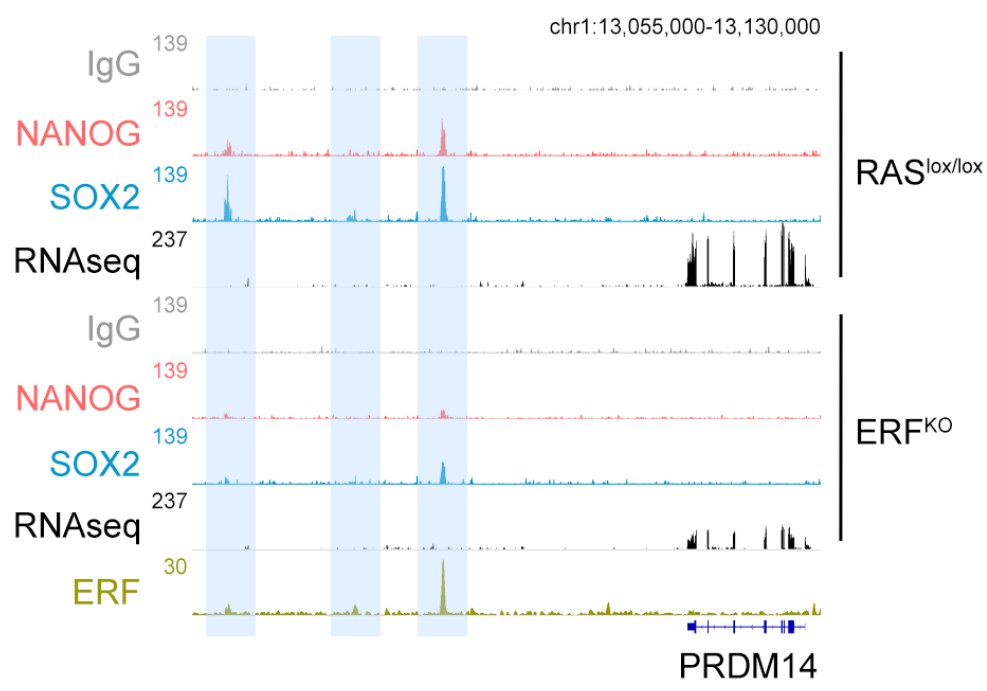


1009 Fig. 4. ERF ensures optimal naïve pluripotent transcription factor expression in ESC. (A) ChIPseq

1010 read density plot (RPKM) showing OCT4, SOX2 and NANOG occupancy at 2074 ERF-binding sites

1011 at enhancers (pink) or 2074 randomly selected non-ERF bound enhancers (orange). Graphs show

1012 quantifications of the TF enrichment in each set of sites. ${ }^{* * *}=p<0.001, \mathrm{~T}$-student. Data was

1013 obtained from (43). (B) Graph showing relative fold change (log2) expression of the indicated

1014 genes in RAS ${ }^{\text {lox/lox }}$ and ERF ${ }^{\mathrm{KO}}$ ESC grown in 2iL. Genes showing at least a 50\% reduction are

1015 highlighted. For each gene, data was normalized to the average across all samples. $* * *=p<0.001$;

$1016 * *=p<0.01$. T-student. Data is shown as triplicates. (C) Graph showing the relative fold change

1017 ( $\log 2)$ expression of a subset of 15 naïve associated genes (Klf4, Esrrb, Tfcp2/1, Tbx3, KIf2, Elf3,

1018 Klf8, Nanog, Prdm14, Tcl1, Zfp42, Nrob1, Prmd16, Dazl and Crxos) in the different genotypes in

1019 2iL conditions. For each gene, data was normalized to the average across all samples. ${ }^{* * *}=$

$1020 \mathrm{p}<0.001$. T-student. (D) Unidimensional PCA plot of RNAseq data of RAS ${ }^{\text {lox/lox }}$ and ERF ${ }^{\mathrm{KO}}$ ESC 1021 cultured in naïve conditions (2iL) or induced to differentiate (FA) during 48 hours to EpiLSC. Three

1022 replicates per condition are shown. PC1 separates samples by differentiation status. (E) Cut\&Run

1023 read density plot (RPKM) showing NANOG and SOX2 occupancy in the set of 2074 ERF-binding

1024 sites at enhancers in RAS ${ }^{\text {lox/lox }}$ (blue) and ERF ${ }^{\mathrm{KO}}$ (green) ESC cultured in 2iL. Corresponding inputs

1025 (IgG) are also shown as reference control. (F) Genome browser tracks showing NANOG and SOX2

1026 occupancy and RNAseq RPKM read count at the PRDM14 gene in the indicated genotypes. ERF

1027 binding profile in RAS ${ }^{\mathrm{KO}}$ ESC is also shown. Inputs (IgG) are also shown as a reference control. ERF

1028 binding sites are highlighted.

1029

1030

1031

1032

1033

1034

1035

1036 
A

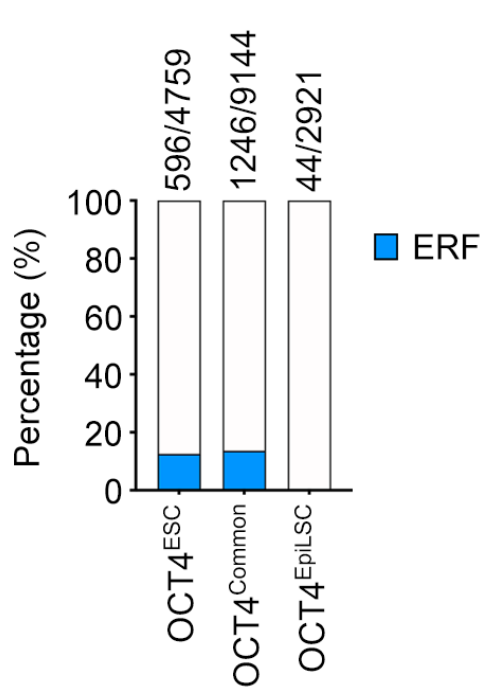

C

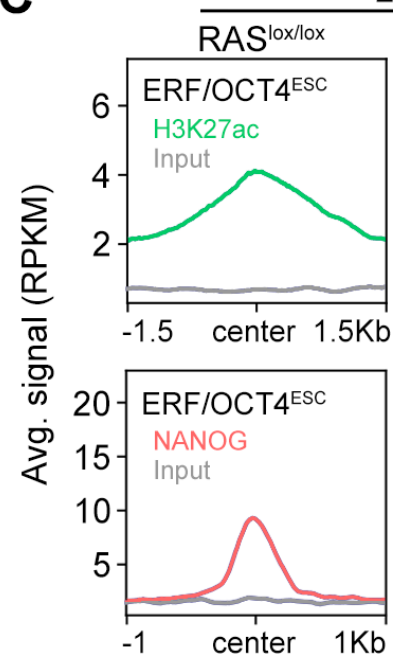

2iL

$\mathrm{RAS}^{\mathrm{KO}}$

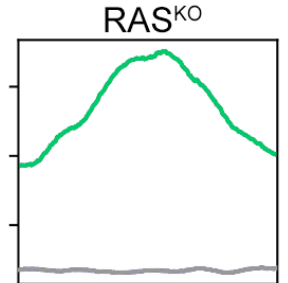

-1.5 center $1.5 \mathrm{~Kb}-1.5$ center $1.5 \mathrm{~Kb}-1.5$ center $1.5 \mathrm{~Kb}$

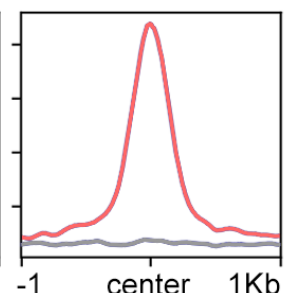

FA

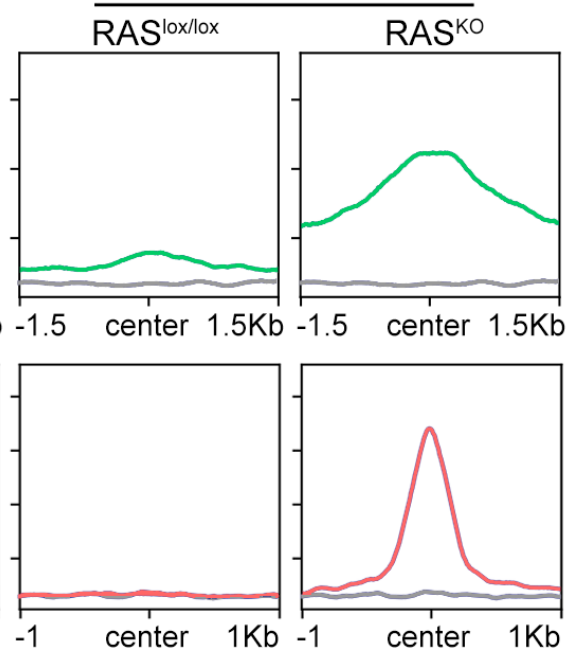

B

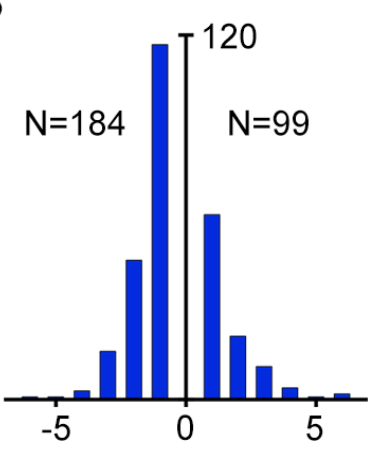

Log2 fold change

RAS ${ }^{10 x / 10 x}$

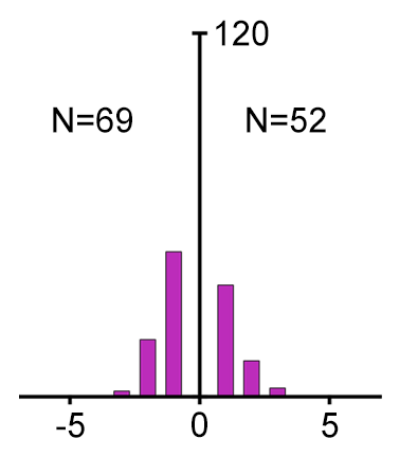

Log2 fold change

O RASKO
E

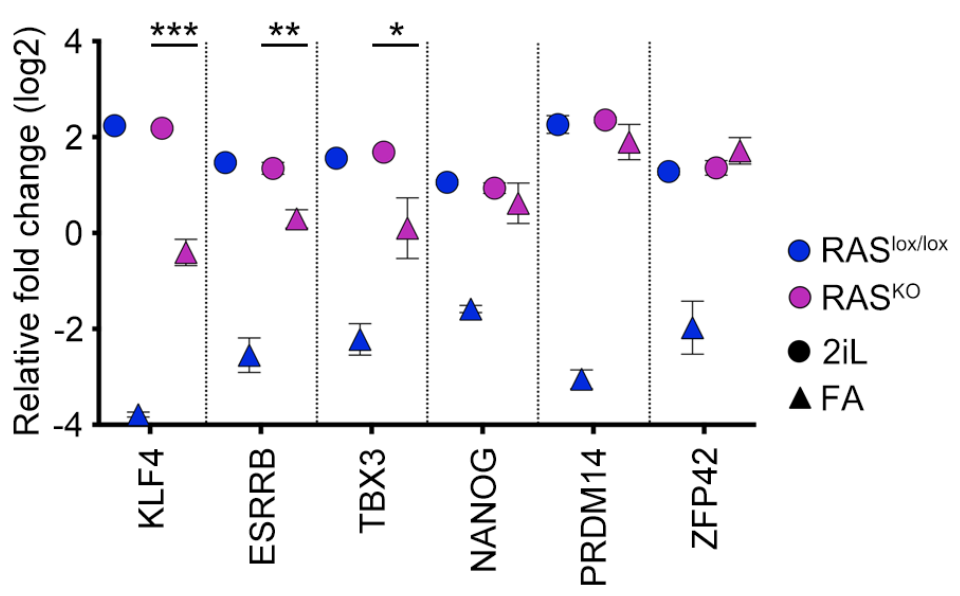

D
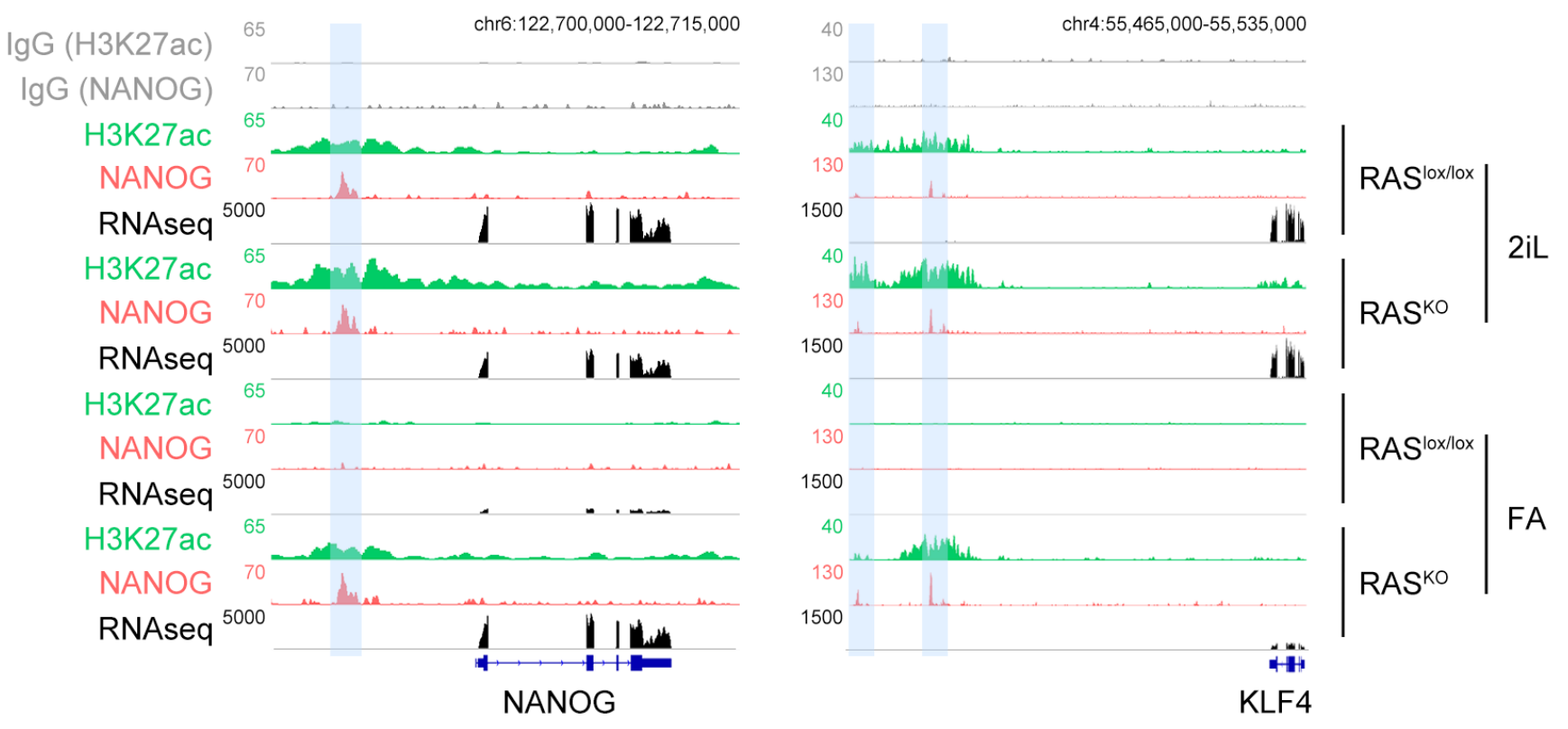

Figure 5 
1037 Fig. 5: The naïve pluripotent transcription factor network is active in FA-RAS ${ }^{\mathrm{KO}}$ ESC. (A) Plot

1038 showing the percentage of OCT4 binding sites co-occupied by ERF in ESC, EpiLSC and common

1039 between ESC and EpiLSC. (B) Histogram plots showing fold expression changes (log2) for genes

1040 associated to ERF/OCT4 $4^{\mathrm{ESC}}$ that are characterized by differential expression between ESC and

1041 EpiLSC in RAS ${ }^{\text {lox/lox }}$ and RAS ${ }^{\text {KO }}$ cells. $\mathrm{N}$ indicates the total number of genes that are up or

1042 downregulated. Genes were associated at every binding site by proximity using GREAT (ref PMID:

1043 20436461). (C) Cut\&Run read density plot (RPKM) showing H3K27ac (plots above, green) and

1044 NANOG (plots below, red) occupancy in ERF/OCT4 ${ }^{\text {ESC }}$ sites in RAS ${ }^{\text {lox/lox }}$ and RAS ${ }^{\text {KO }}$ cultured in 2iL

1045 or differentiated to EpiLSC (FA). Corresponding inputs (IgG) are also shown as reference control.

1046 (D) Genome browser tracks showing H3K27ac deposition, NANOG occupancy and RNAseq RPKM

1047 read count at the KLF4 and NANOG genes in the indicated genotypes. Inputs (IgG) are also shown

1048 as a reference control. (E) Graph showing the relative fold change ( $\log 2)$ expression of the 1049 indicated ERF-bound super-enhancer associated genes in RAS ${ }^{\text {lox/lox }}$ and RAS ${ }^{\mathrm{KO}}$ cultured in 2iL or

1050 differentiated to EpiLSC (FA). Genes showing at least a 50\% reduction are highlighted. For each

1051 gene, data was normalized to the average across all samples. ${ }^{* * *}=p<0.001 ; * *=p<0.01 ;^{*}=<0.05$

1052 T-student. Data shown are averages from triplicates.

1053

1054

1055

1056

1057

1058

1059

1060

1061

1062

1063

1064

1065 
A

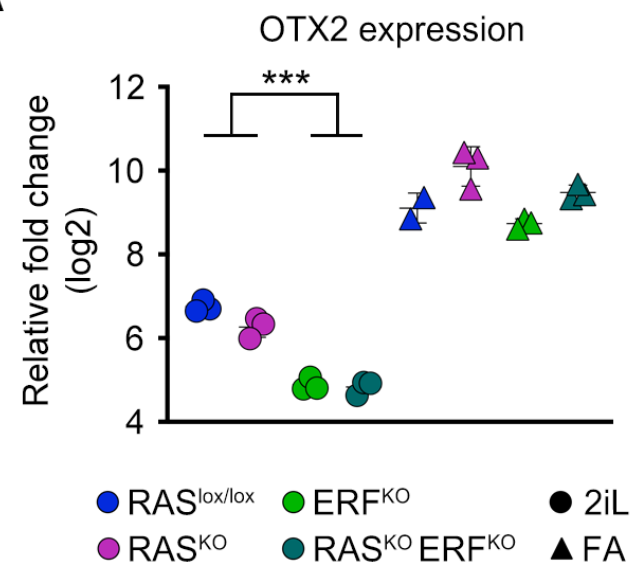

B

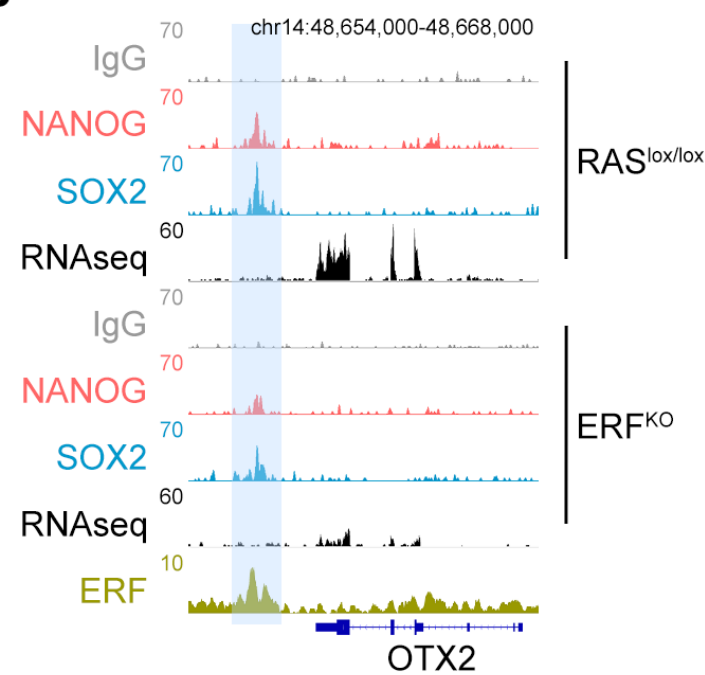

C

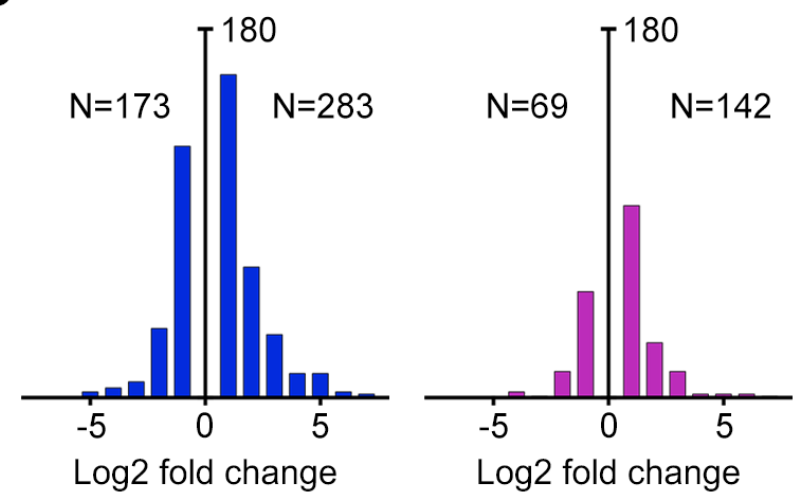

RAS $10 x / 10 x$

RASKO
D

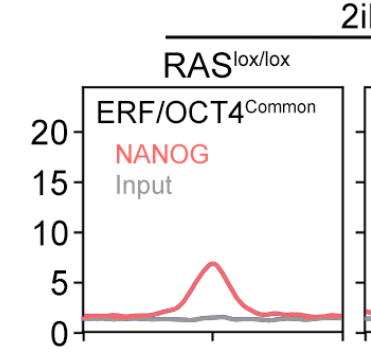

2iL
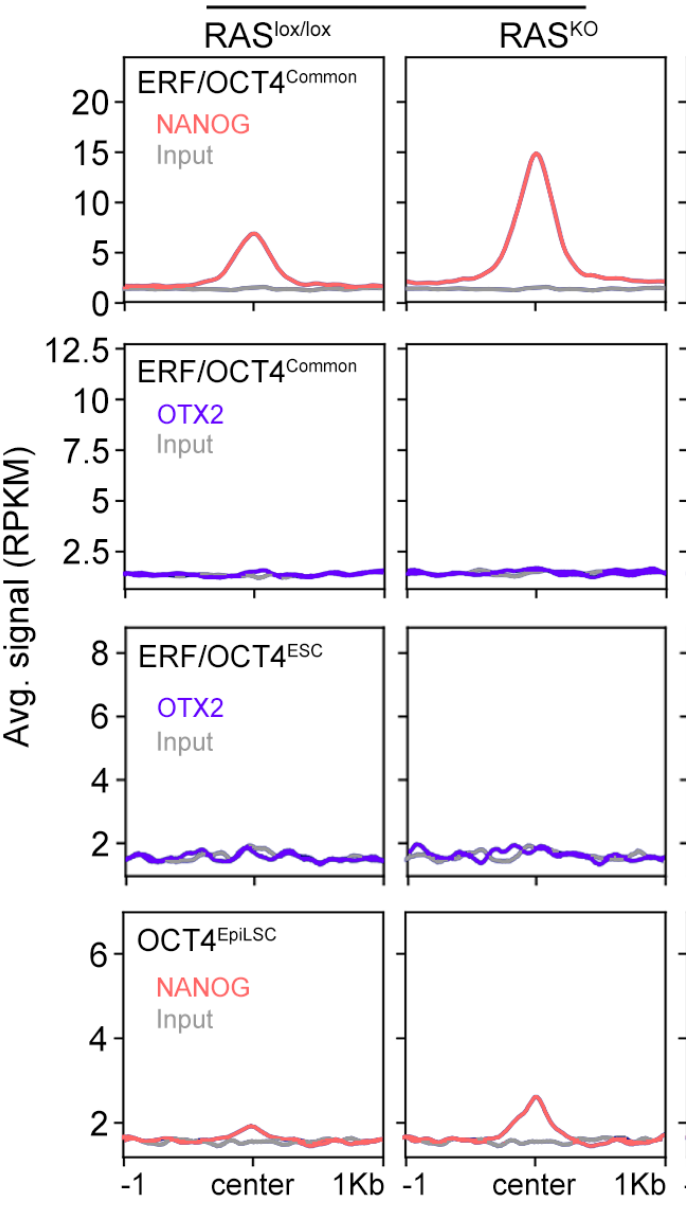

E

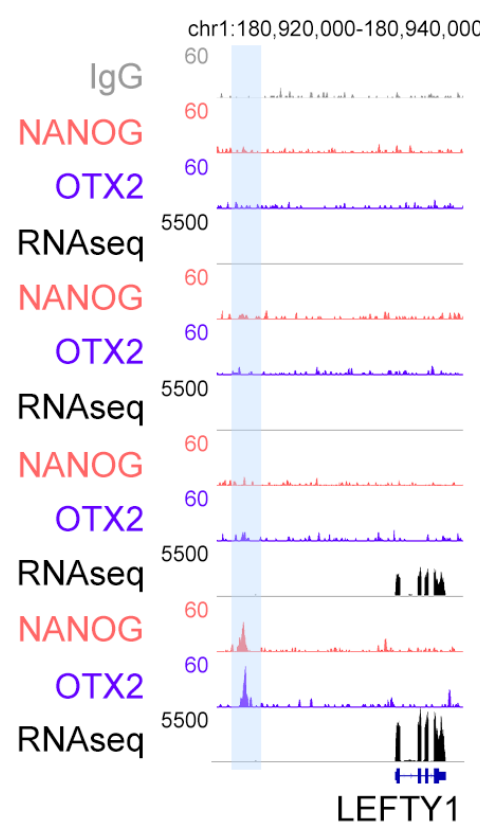

FA
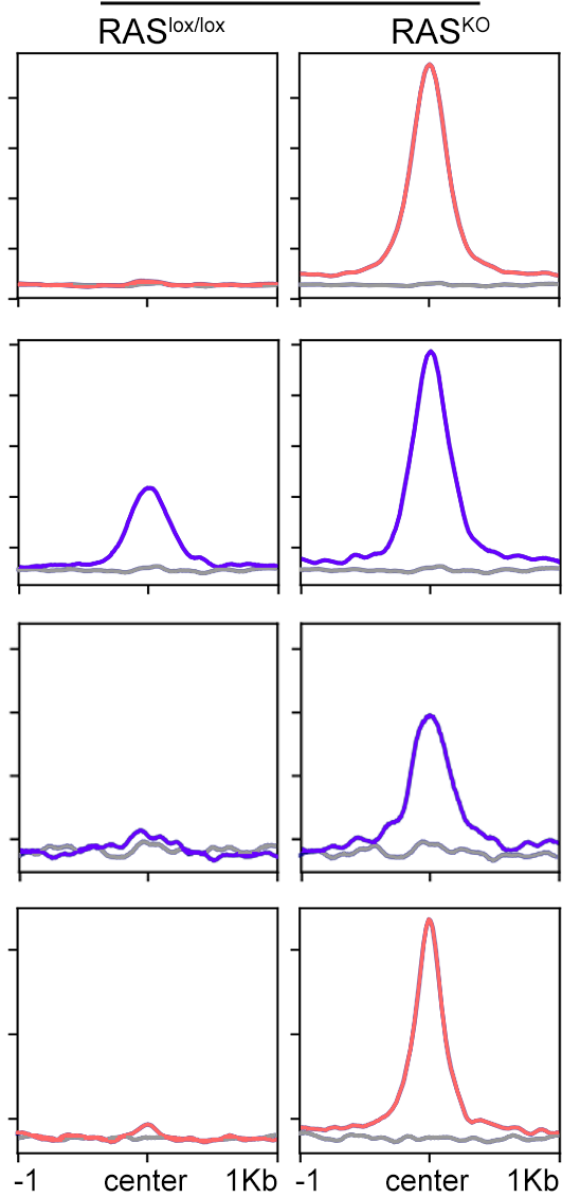

chr1:180,888,000-180,900,000

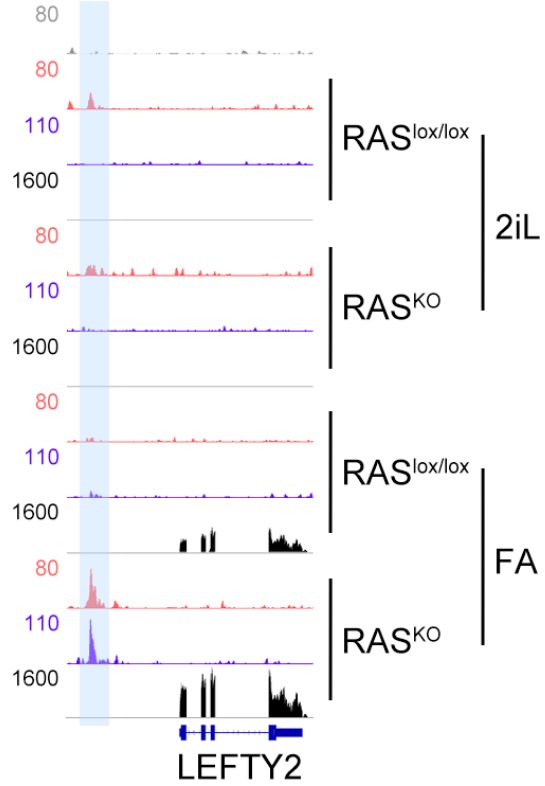


1066 Fig. 6. OTX2 shows promiscuous binding in naïve and primed genes in FA-RAS ${ }^{\mathrm{KO}}$ ESC. (A) Plot

1067 showing the relative fold change ( $\log 2)$ expression for OTX2 in ESC from all genotypes cultured in

10682 iL or differentiated to EpiLSC (FA). Data was normalized to the average across all samples. ${ }^{* * *}=$

$1069 \mathrm{p}<0.001 ;$ T-student. Data shown from triplicates. (B) Genome browser tracks showing SOX2 and

1070 NANOG occupancy and RNAseq RPKM read count at the OTX2 gene in RAS ${ }^{\text {lox/lox }}$ and ERF ${ }^{\mathrm{KO}}$ ESC

1071 cultured in 2iL. ERF binding profile in RAS ${ }^{K O}$ ESC is also shown. Inputs (IgG) are also shown as a

1072 reference control. ERF binding sites are highlighted. (C) Histogram plots showing fold expression

1073 changes $(\log 2)$ for genes associated to ERF/OCT4 $4^{\text {Common }}$ that showed differential expression

1074 between ESC and EpiLSC in RAS ${ }^{\text {lox/lox }}$ and RAS ${ }^{\mathrm{KO}}$ cells. $\mathrm{N}$ indicates the total number of genes that

1075 are up or downregulated. Genes were associated at every binding site by proximity using GREAT

1076 (ref PMID: 20436461). (D) Cut\&Run read density plot (RPKM) showing NANOG (red) and OTX2

1077 (purple) occupancy in the indicated ERF/OCT4 ${ }^{\text {Common, ERF/OCT4 }}{ }^{\text {ESC }}$ and ERF/OCT4 ${ }^{\text {EpiLSC }}$ sites in 1078 RAS $^{\text {lox/lox }}$ and RAS ${ }^{\text {KO }}$ ESC cultured in 2iL or differentiated to EpiLSC (FA). Corresponding inputs (IgG)

1079 are also shown as reference control. (E) Genome browser tracks showing NANOG and OTX2 1080 occupancy and RNAseq RPKM read count at the LEFTY1 and LEFTY2 genes in RAS lox/lox and RAS ${ }^{\text {KO }}$

1081 ESC cultured in 2iL or differentiated to EpiLSC (FA). Inputs (IgG) are also shown as a reference 1082 control. Blue squares showed ERF binding sites.

1083

1084

1085

1086

1087

1088

1089

1090

1091

1092

1093 
A
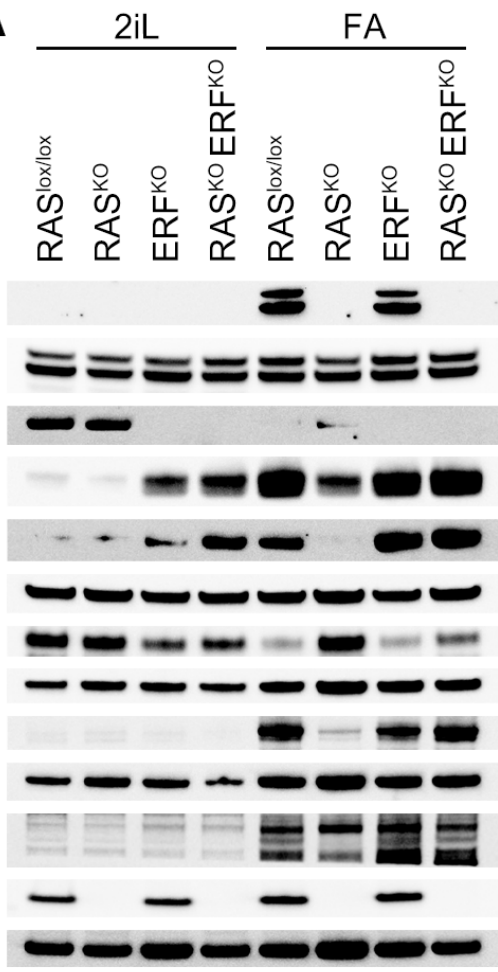

pERK

ERK

ERF

LIN28A

LIN28B

TUBULIN

NANOG

TUBULIN

DNMT3B

TUBULIN

DNMT3A

PanRAS

TUBULIN
B

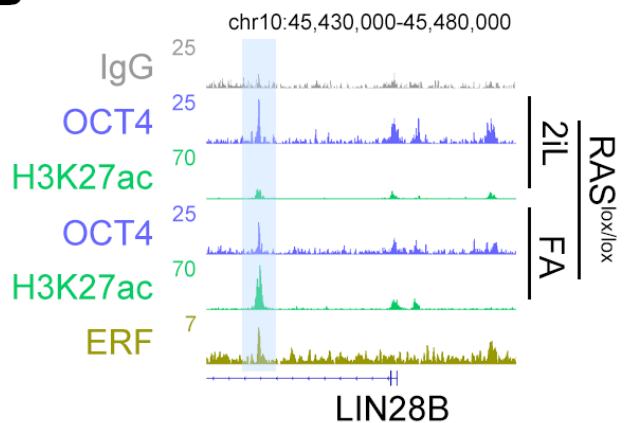

D

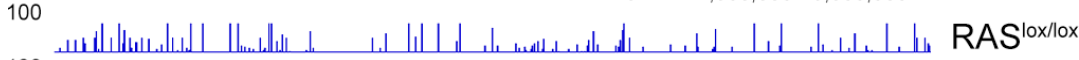
100

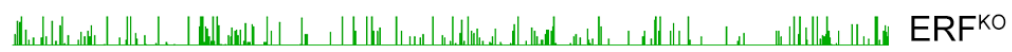

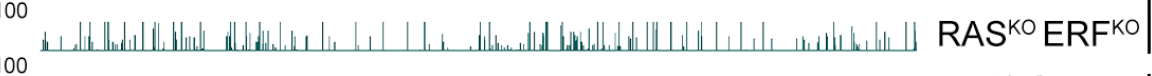
每

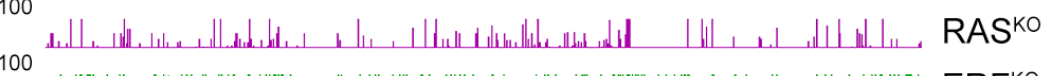
100 ERF

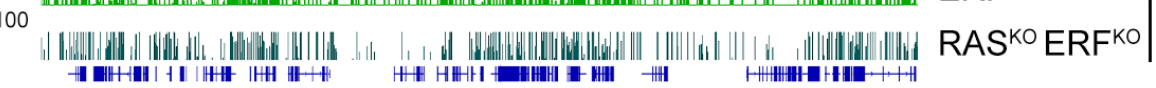

E

2iL

FA

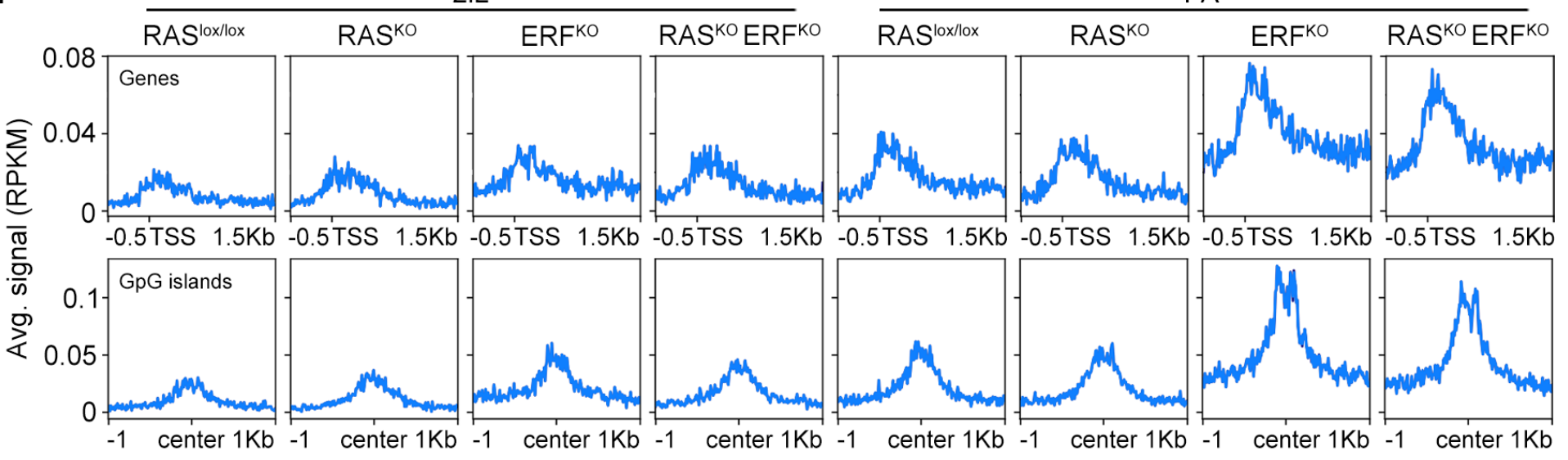

Naïve ESC

FA-RAS ${ }^{\text {KO }}$
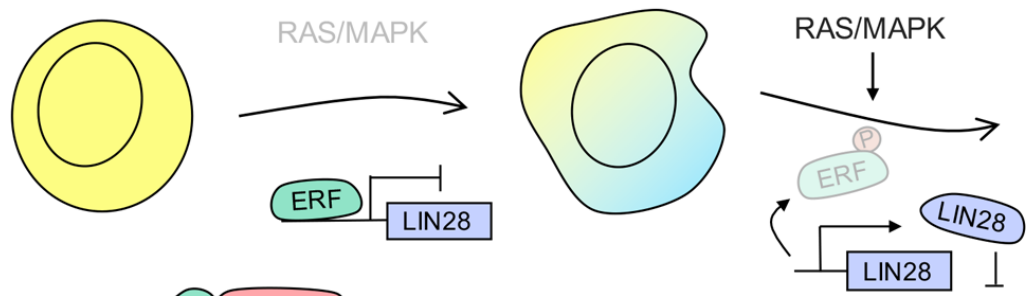

Epiblast-like Cells

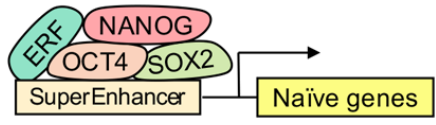

Naïve genes 
1094 Fig. 7. ERF controls the levels of de novo methylation during transition to EpiLSC. (A) Western

1095 blot analysis of the indicated proteins performed in ESC from all genotypes cultured in 2iL or

1096 differentiated to EpiLSC (FA). Tubulin levels for the corresponding blots are shown as a loading

1097 control. One representative experiment is shown but at least two independent clones per

1098 condition and genotype were used. (B) Genome browser tracks showing H3K27ac deposition and

1099 OCT4 occupancy at the LIN28B gene in RAS ${ }^{\text {lox/lox }}$ ESC cultured in 2iL or differentiated to EpiLSC

1100 (FA). ERF binding profile in RAS ${ }^{\mathrm{KO}}$ ESC is also shown. Inputs (IgG) are also shown as a reference

1101 control. ERF binding sites are highlighted. (C) Graph showing the percentage of methylated CpG

1102 sites identified by RRBS in ESC from all genotypes cultured in 2iL or differentiated to EpiLSC (FA).

1103 (D) Genome browser tracks showing as a representative example the level of methylation at CpG

1104 sites in a region of chromosome 12 in ESC from all genotypes cultured in 2iL or differentiated to

1105 EpiLSC (FA). (E) \%CpG methylation averaged across the TSS of all protein-coding mouse genes

1106 (upper panels) or centered at CpG islands (lower panels) in ESC from all genotypes cultured in 2iL

1107 or differentiated to EpiLSC (FA). (F) Schematic model showing the dual role of ERF during the 1108 naïve to primed pluripotent transition. In the absence of FGF signaling, ERF ensures an optimal

1109 level of expression for naïve transcription factors. Following ERF phosphorylation and gene 1110 silencing, ESC are able to exit and transition into EpiLSC along with global CpG methylation and 1111 silencing of the naïve transcriptional network.

1112

1113 